ESAIM: COCV 20 (2014) 704-724

DOI: $10.1051 / \mathrm{cocv} / 2013080$
ESAIM: Control, Optimisation and Calculus of Variations

www.esaim-cocv.org

\title{
SECOND-ORDER SUFFICIENT CONDITIONS FOR STRONG SOLUTIONS TO OPTIMAL CONTROL PROBLEMS*
}

\author{
J. Frédéric Bonnans ${ }^{1}$, Xavier Dupuis ${ }^{1}$ and Laurent Pfeiffer ${ }^{1}$
}

\begin{abstract}
In this article, given a reference feasible trajectory of an optimal control problem, we say that the quadratic growth property for bounded strong solutions holds if the cost function of the problem has a quadratic growth over the set of feasible trajectories with a bounded control and with a state variable sufficiently close to the reference state variable. Our sufficient second-order optimality conditions in Pontryagin form ensure this property and ensure a fortiori that the reference trajectory is a bounded strong solution. Our proof relies on a decomposition principle, which is a particular second-order expansion of the Lagrangian of the problem.
\end{abstract}

Mathematics Subject Classification. 49K15, 34K35, 90C48.

Received May 23, 2013. Revised September 16, 2013.

Published online March 14, 2014.

\section{INTRODUCTION}

In this paper, we consider an optimal control problem with final-state constraints, pure state constraints, and mixed control-state constraints. Given a feasible control $\bar{u}$ and its associated state variable $\bar{y}$, we give secondorder conditions ensuring that for all $R>\|\bar{u}\|_{\infty}$, there exist $\varepsilon>0$ and $\alpha>0$ such that for all feasible trajectory $(u, y)$ with $\|u\|_{\infty} \leq R$ and $\|y-\bar{y}\|_{\infty} \leq \varepsilon$,

$$
J(u, y)-J(\bar{u}, \bar{y}) \geq \alpha\left(\|u-\bar{u}\|_{2}^{2}+\left|y_{0}-\bar{y}_{0}\right|^{2}\right),
$$

where $J(u, y)$ is the cost function to minimize. We call this property quadratic growth for bounded strong solutions. Its specificity lies in the fact that the quadratic growth is ensured for controls which may be far from $\bar{u}$ in $L^{\infty}$ norm.

Our approach is based on the theory of second-order optimality conditions for optimization problems in Banach spaces $[7,13,15]$. A local optimal solution satisfies first- and second-order necessary conditions; denoting by $\Omega$ the Hessian of the Lagrangian, theses conditions state that under the extended polyhedricity condition [6], Section 3.2, the supremum of $\Omega$ over the set of Lagrange multipliers is nonnegative for all critical directions.

\footnotetext{
Keywords and phrases. Optimal control, second-order sufficient conditions, quadratic growth, bounded strong solutions, Pontryagin multipliers, pure state and mixed control-state constraints, decomposition principle.

* The research leading to these results has received funding from the EU 7th Framework Programme (FP7- PEOPLE-2010ITN), under GA number 264735-SADCO, and from the Gaspard Monge Program for Optimization and operations research (PGMO).

1 Inria Saclay and CMAP, Ecole Polytechnique. Route de Saclay, 91128 Palaiseau Cedex, France. frederic.bonnans@inria.fr; xavier.dupuis@cmap.polytechnique.fr; laurent.pfeiffer@polytechnique.edu
} 
If the supremum of $\Omega$ is positive for nonzero critical directions, we say that the second-order sufficient optimality conditions hold and under some assumptions, a quadratic growth property is then satisfied. This approach can be used for optimal control problems with constraints of any kind. For example, Stefani and Zezza [22] dealt with problems with mixed control-state equality constraints and Bonnans and Hermant [4] with problems with pure state and mixed control-state constraints. However, the quadratic growth property which is then satisfied holds for controls which are sufficiently close to $\bar{u}$ in uniform norm and only ensures that $(\bar{u}, \bar{y})$ is a weak solution.

For Pontryagin minima, that is to say minima locally optimal in a $L^{1}$ neighborhood of $\bar{u}$, the necessary conditions can be strengthened. The first-order conditions are nothing but the well-known Pontryagin's principle, historically formulated in [21] and extended to problems with various constraints by many authors, such as Hestenes for problems with mixed control-state constraints [11], Dubovitskii and Milyutin for problems with pure state and mixed control-state constraints in early Russian references [9,10], as highlighted by Dmitruk [8]. We refer to the survey by Hartl et al. for more references on this principle.

We say that the second-order necessary conditions are in Pontryagin form if the supremum of $\Omega$ is taken over the set of Pontryagin multipliers, these multipliers being the Lagrange multipliers for which Pontryagin's principle holds; under some hypotheses, these conditions are satisfied for Pontryagin minima. The sufficient conditions in Pontryagin form are as follows: the supremum of $\Omega$ over Pontryagin multipliers only is positive for nonzero critical directions and for all bounded neighborhood of $\bar{u}$, there exists a Pontryagin multiplier which is such the Hamiltonian has itself a quadratic growth; if these conditions hold, then the quadratic growth for bounded strong solutions holds.

Osmolovskii proved the necessary conditions in [17] and gave sufficient conditions for the quadratic growth for bounded strong solutions in [18], for problems with final-state constraints, mixed constraints and possibly discontinuous optimal controls. These results had been announced in [16]. The quadratic growth property which is established in [18] is more general than ours: it involves the so-called violation function (which is equal to the difference of cost for a feasible variation) and a general class of cost functionals for the growth property. A simplified version of this article was published by the same author, in which the optimal control is continuous [19]. In [20], Maurer and Osmolovskii considered the simpler case of final-state constraints and mixed equality constraints.

For problems with pure and mixed inequality constraints, we proved the second-order necessary conditions in Pontryagin form [2]; in the present paper, we prove that the sufficient conditions in Pontryagin form ensure the quadratic growth property for bounded strong solutions. Our proof is based on an extension of the decomposition principle of Bonnans and Osmolovskii [5] to the constrained case. This principle is a particular second-order expansion of the Lagrangian, which takes into account the fact that the control may have large perturbations in uniform norm. Note that the difficulties arising in the extension of the principle and the proof of quadratic growth are mainly due to the presence of mixed control-state constraints.

In this article, we do not need the independence of the gradients of active mixed constraints, an assumption needed in the aforementioned papers, instead, we only use the inward condition. We also use the strengthened Legendre condition, which holds if the Hessian of the augmented Hamiltonian with respect to the control is uniformly positive. This is a strong assumption; when there are mixed constraints (or simply control constraints), this assumption cannot be considered as a natural strengthening of the necessary conditions. However, it simplifies considerably the proof of quadratic growth. Note that it is not required in the decomposition principle. Note also that Osmolovskii proved the quadratic growth without this assumption in [18] and used instead estimates of the distance to the critical cone which were obtained with generalizations of Hoffman's lemma.

The outline of the paper is as follows. In Section 2, we set our optimal control problem. Section 3 is devoted to technical aspects related to the reduction of state constraints. We prove the decomposition principle in Section 4 (Thm. 4.2) and prove the quadratic growth property for bounded strong solutions in Section 5 (Thm. 5.4). In Section 6 , we prove that under technical assumptions, the sufficient conditions are not only sufficient but also necessary to ensure the quadratic growth property (Thm. 6.3). 


\section{Notations.}

For a function $h$ that depends only on time $t$, we denote by $h_{t}$ its value at time $t$, by $h_{i, t}$ the value of its $i$ th component if $h$ is vector-valued, and by $\dot{h}$ its derivative. For a function $h$ that depends on $(t, x)$, we denote by $D_{t} h$ and $D_{x} h$ its partial derivatives. We use the symbol $D$ without any subscript for the differentiation w.r.t. all variables except $t, e . g . D h=D_{(u, y)} h$ for a function $h$ that depends on $(t, u, y)$. We use the same convention for higher order derivatives.

We identify the dual space of $\mathbb{R}^{n}$ with the space $\mathbb{R}^{n *}$ of $n$-dimensional horizontal vectors. Generally, we denote by $X^{*}$ the dual space of a topological vector space $X$. Given a convex subset $K$ of $X$ and a point $x$ of $K$, we denote by $T_{K}(x)$ and $N_{K}(x)$ the tangent and normal cone to $K$ at $x$, respectively; see ([6], Sect. 2.2.4) for their definition.

We denote by $|\cdot|$ both the Euclidean norm on finite-dimensional vector spaces and the cardinal of finite sets, and by $\|\cdot\|_{s}$ and $\|\cdot\|_{q, s}$ the standard norms on the Lesbesgue spaces $L^{s}$ and the Sobolev spaces $W^{q, s}$, respectively.

We denote by $B V([0, T])$ the space of functions of bounded variation on the closed interval $[0, T]$. Any $h \in B V([0, T])$ has a derivative $\mathrm{d} h$ which is a finite Radon measure on $[0, T]$ and $h_{0}$ (resp. $\left.h_{T}\right)$ is defined by $h_{0}:=h_{0_{+}}-\mathrm{d} h(0)$ (resp. $h_{T}:=h_{T_{-}}+\mathrm{d} h(T)$ ). Thus $B V([0, T])$ is endowed with the following norm: $\|h\|_{B V}:=\|\mathrm{d} h\|_{\mathcal{M}}+\left|h_{T}\right|$. See ([1], Sect. 3.2) for a rigorous presentation of $B V$.

All vector-valued inequalities have to be understood coordinate-wise.

\section{Setting}

\subsection{The optimal control problem}

We formulate in this section the optimal control problem under study and we use the same framework as in [2]. We refer to this article for supplementary comments on the different assumptions made. Consider the state equation

$$
\dot{y}_{t}=f\left(t, u_{t}, y_{t}\right) \quad \text { for a.a. } t \in(0, T) .
$$

Here, $u$ is a control which belongs to $\mathcal{U}, y$ is a state which belongs to $\mathcal{Y}$, where

$$
\mathcal{U}:=L^{\infty}\left(0, T ; \mathbb{R}^{m}\right), \quad \mathcal{Y}:=W^{1, \infty}\left(0, T ; \mathbb{R}^{n}\right),
$$

and $f:[0, T] \times \mathbb{R}^{m} \times \mathbb{R}^{n} \rightarrow \mathbb{R}^{n}$ is the dynamics. Given $y^{0} \in \mathbb{R}^{n}$ and $u \in \mathcal{U}$, we denote by $y\left[u, y^{0}\right]$ the solution to (2.1) with the initial condition $y_{0}=y^{0}$. Consider constraints of various types on the system: the mixed control-state constraints, or mixed constraints

$$
c\left(t, u_{t}, y_{t}\right) \leq 0 \quad \text { for a.a. } t \in(0, T),
$$

the pure state constraints, or state constraints

$$
g\left(t, y_{t}\right) \leq 0 \quad \text { for a.a. } t \in(0, T),
$$

and the initial-final state constraints

$$
\left\{\begin{array}{c}
\Phi^{E}\left(y_{0}, y_{T}\right)=0 \\
\Phi^{I}\left(y_{0}, y_{T}\right) \leq 0 .
\end{array}\right.
$$

Here $c:[0, T] \times \mathbb{R}^{m} \times \mathbb{R}^{n} \rightarrow \mathbb{R}^{n_{c}}, g:[0, T] \times \mathbb{R}^{n} \rightarrow \mathbb{R}^{n_{g}}, \Phi^{E}: \mathbb{R}^{n} \times \mathbb{R}^{n} \rightarrow \mathbb{R}^{n_{\Phi} E}, \Phi^{I}: \mathbb{R}^{n} \times \mathbb{R}^{n} \rightarrow \mathbb{R}^{n_{\Phi} I}$. Finally, consider the cost function $\phi: \mathbb{R}^{n} \times \mathbb{R}^{n} \rightarrow \mathbb{R}$. The optimal control problem is then

$$
\min _{(u, y) \in \mathcal{U} \times \mathcal{Y}} \phi\left(y_{0}, y_{T}\right) \quad \text { subject to } \quad(2.1)-(2.5) .
$$

We call a trajectory any pair $(u, y) \in \mathcal{U} \times \mathcal{Y}$ such that (2.1) holds. We say that a trajectory is feasible for problem $(P)$ if it satisfies constraints $(2.3)-(2.5)$, and denote by $F(P)$ the set of feasible trajectories. From now on, we fix a feasible trajectory $(\bar{u}, \bar{y})$. 
Similarly to ([22], Def. 2.1), we introduce the following Carathéodory-type regularity notion:

Definition 2.1. We say that $\varphi:[0, T] \times \mathbb{R}^{m} \times \mathbb{R}^{n} \rightarrow \mathbb{R}^{s}$ is uniformly quasi- $C^{k}$ iff

(i) for a.a. $t,(u, y) \mapsto \varphi(t, u, y)$ is of class $C^{k}$, and the modulus of continuity of $(u, y) \mapsto D^{k} \varphi(t, u, y)$ on any compact of $\mathbb{R}^{m} \times \mathbb{R}^{n}$ is uniform w.r.t. $t$.

(ii) for $j=0, \ldots, k$, for all $(u, y), t \mapsto D^{j} \varphi(t, u, y)$ is essentially bounded.

Remark 2.2. If $\varphi$ is uniformly quasi- $C^{k}$, then $D^{j} \varphi$ for $j=0, \ldots, k$ are essentially bounded on any compact, and $(u, y) \mapsto D^{j} \varphi(t, u, y)$ for $j=0, \ldots, k-1$ are locally Lipschitz, uniformly w.r.t. $t$.

The regularity assumption that we need for the quadratic growth property is the following:

Assumption 1. The mappings $f, c$ and $g$ are uniformly quasi- $C^{2}, g$ is differentiable, $D_{t} g$ is uniformly quasi- $C^{1}$, $\Phi^{E}, \Phi^{I}$, and $\phi$ are $C^{2}$.

Note that this assumption will be strengthened in Section 6 .

Definition 2.3. We say that the inward condition for the mixed constraints holds iff there exist $\gamma>0$ and $\bar{v} \in \mathcal{U}$ such that

$$
c\left(t, \bar{u}_{t}, \bar{y}_{t}\right)+D_{u} c\left(t, \bar{u}_{t}, \bar{y}_{t}\right) \bar{v}_{t} \leq-\gamma, \quad \text { for a.a. } t .
$$

In the sequel, we will always make the following assumption:

Assumption 2. The inward condition for the mixed constraints holds.

Assumption 2 ensures that the component of the Lagrange multipliers associated with the mixed constraints belongs to $L^{\infty}\left(0, T ; \mathbb{R}^{n_{c} *}\right)$, see e.g. ([5], Thm. 3.1). This assumption will also play a role in the decomposition principle.

\subsection{Bounded strong optimality and quadratic growth}

Let us introduce various notions of minima, following [16].

Definition 2.4. We say that $(\bar{u}, \bar{y})$ is a bounded strong minimum iff for any $R>\|\bar{u}\|_{\infty}$, there exists $\varepsilon>0$ such that

$$
\begin{aligned}
\phi\left(\bar{y}_{0}, \bar{y}_{T}\right) \leq \phi\left(y_{0}, y_{T}\right), & \text { for all }(u, y) \in F(P) \text { such that } \\
& \|y-\bar{y}\|_{\infty} \leq \varepsilon \text { and }\|u\|_{\infty} \leq R,
\end{aligned}
$$

a Pontryagin minimum iff for any $R>\|\bar{u}\|_{\infty}$, there exists $\varepsilon>0$ such that

$$
\phi\left(\bar{y}_{0}, \bar{y}_{T}\right) \leq \phi\left(y_{0}, y_{T}\right), \quad \begin{aligned}
& \text { for all }(u, y) \in F(P) \text { such that } \\
& \\
& \|u-\bar{u}\|_{1}+\|y-\bar{y}\|_{\infty} \leq \varepsilon \text { and }\|u\|_{\infty} \leq R,
\end{aligned}
$$

a weak minimum iff there exists $\varepsilon>0$ such that

$$
\begin{array}{ll}
\phi\left(\bar{y}_{0}, \bar{y}_{T}\right) \leq \phi\left(y_{0}, y_{T}\right), & \text { for all }(u, y) \in F(P) \text { such that } \\
& \|u-\bar{u}\|_{\infty}+\|y-\bar{y}\|_{\infty} \leq \varepsilon
\end{array}
$$

Obviously, $(2.7) \Rightarrow(2.8) \Rightarrow(2.9)$.

Definition 2.5. We say that the quadratic growth property for bounded strong solutions holds at $(\bar{u}, \bar{y})$ iff for all $R>\|\bar{u}\|_{\infty}$, there exist $\varepsilon_{R}>0$ and $\alpha_{R}>0$ such that for all feasible trajectory $(u, y)$ satisfying $\|u\|_{\infty} \leq R$ and $\|y-\bar{y}\|_{\infty} \leq \varepsilon_{R}$,

$$
\phi\left(y_{0}, y_{T}\right)-\phi\left(\bar{y}_{0}, \bar{y}_{T}\right) \geq \alpha_{R}\left(\left|y_{0}-\bar{y}_{0}\right|^{2}+\|u-\bar{u}\|_{2}^{2}\right) .
$$

The goal of the article is to characterize this property. If it holds at $(\bar{u}, \bar{y})$, then $(\bar{u}, \bar{y})$ is a bounded strong solution to the problem. 


\subsection{Multipliers}

We define the Hamiltonian and the augmented Hamiltonian respectively by

$$
H[p](t, u, y):=p f(t, u, y), \quad H^{a}[p, \nu](t, u, y):=p f(t, u, y)+\nu c(t, u, y),
$$

for $(p, \nu, t, u, y) \in \mathbb{R}^{n *} \times \mathbb{R}^{n_{c} *} \times[0, T] \times \mathbb{R}^{m} \times \mathbb{R}^{n}$. We define the end points Lagrangian by

$$
\Phi[\beta, \Psi]\left(y_{0}, y_{T}\right):=\beta \phi\left(y_{0}, y_{T}\right)+\Psi \Phi\left(y_{0}, y_{T}\right),
$$

for $\left(\beta, \Psi, y_{0}, y_{T}\right) \in \mathbb{R} \times \mathbb{R}^{n_{\Phi} *} \times \mathbb{R}^{n} \times \mathbb{R}^{n}$, where $n_{\Phi}=n_{\Phi^{E}}+n_{\Phi^{I}}$ and $\Phi=\left(\begin{array}{c}\Phi^{E} \\ \Phi^{I}\end{array}\right)$.

We set

$$
K_{c}:=L^{\infty}\left(0, T ; \mathbb{R}_{-}^{n_{c}}\right), \quad K_{g}:=C\left([0, T] ; \mathbb{R}_{-}^{n_{g}}\right), \quad K_{\Phi}:=\{0\}_{\mathbb{R}^{n_{\Phi} E}} \times \mathbb{R}_{-}^{n_{\Phi} I},
$$

so that the constraints $(2.3)-(2.5)$ can be rewritten as

$$
c(\cdot, u, y) \in K_{c}, \quad g(\cdot, y) \in K_{g}, \quad \Phi\left(y_{0}, y_{T}\right) \in K_{\Phi} .
$$

Recall that the dual space of $C\left([0, T] ; \mathbb{R}^{n_{g}}\right)$ is the space $\mathcal{M}\left([0, T] ; \mathbb{R}^{n_{g^{*}}}\right)$ of finite vector-valued Radon measures. We denote by $\mathcal{M}\left([0, T] ; \mathbb{R}^{n_{g} *}\right)_{+}$the cone of positive measures in this dual space. Let

$$
E:=\mathbb{R} \times \mathbb{R}^{n_{\Phi *}} \times L^{\infty}\left(0, T ; \mathbb{R}^{n_{c^{*}}}\right) \times \mathcal{M}\left([0, T] ; \mathbb{R}^{n_{g^{*}}}\right) .
$$

Let $N_{K_{c}}(c(\cdot, \bar{u}, \bar{y}))$ be the set of elements in the normal cone to $K_{c}$ at $c(\cdot, \bar{u}, \bar{y})$ that belong to $L^{\infty}\left(0, T ; \mathbb{R}^{n_{c} *}\right)$, i.e.

$$
N_{K_{c}}(c(\cdot, \bar{u}, \bar{y})):=\left\{\nu \in L^{\infty}\left(0, T ; \mathbb{R}_{+}^{n_{c^{*}}}\right): \nu_{t} c\left(t, \bar{u}_{t}, \bar{y}_{t}\right)=0 \text { for a.a. } t\right\} .
$$

Let $N_{K_{g}}(g(\cdot, \bar{y}))$ be the normal cone to $K_{g}$ at $g(\cdot, \bar{y})$, i.e.

$$
N_{K_{g}}(g(\cdot, \bar{y})):=\left\{\mu \in \mathcal{M}\left([0, T] ; \mathbb{R}^{n_{g^{*}}}\right)_{+}: \int_{[0, T]}\left(\mathrm{d} \mu_{t} g\left(t, \bar{y}_{t}\right)\right)=0\right\} .
$$

Let $N_{K_{\Phi}}\left(\Phi\left(\bar{y}_{0}, \bar{y}_{T}\right)\right)$ be the normal cone to $K_{\Phi}$ at $\Phi\left(\bar{y}_{0}, \bar{y}_{T}\right)$, i.e.

$$
N_{K_{\Phi}}\left(\Phi\left(\bar{y}_{0}, \bar{y}_{T}\right)\right):=\left\{\Psi \in \mathbb{R}^{n_{\Phi} *}: \begin{array}{l}
\Psi_{i} \geq 0 \\
\Psi_{i} \Phi_{i}\left(\bar{y}_{0}, \bar{y}_{T}\right)=0
\end{array} \text { for } n_{\Phi^{E}}<i \leq n_{\Phi}\right\} .
$$

Finally, let

$$
N(\bar{u}, \bar{y}):=\mathbb{R}_{+} \times N_{K_{\Phi}}\left(\Phi\left(\bar{y}_{0}, \bar{y}_{T}\right)\right) \times N_{K_{c}}(c(\cdot, \bar{u}, \bar{y})) \times N_{K_{g}}(g(\cdot, \bar{y})) \subset E .
$$

We define the costate space

$$
\mathcal{P}:=B V\left([0, T] ; \mathbb{R}^{n *}\right) .
$$

Given $\lambda=(\beta, \Psi, \nu, \mu) \in E$, we consider the costate equation in $\mathcal{P}$

$$
\left\{\begin{aligned}
-\mathrm{d} p_{t} & =D_{y} H^{a}\left[p_{t}, \nu_{t}\right]\left(t, \bar{u}_{t}, \bar{y}_{t}\right) \mathrm{d} t+\mathrm{d} \mu_{t} D g\left(t, \bar{y}_{t}\right) \\
p_{T} & =D_{y_{T}} \Phi[\beta, \Psi]\left(\bar{y}_{0}, \bar{y}_{T}\right) .
\end{aligned}\right.
$$

Definition 2.6. Let $\lambda=(\beta, \Psi, \nu, \mu) \in E$. We say that the solution of the costate equation $(2.21) p^{\lambda} \in \mathcal{P}$ is an associated costate iff

$$
-p_{0}^{\lambda}=D_{y_{0}} \Phi[\beta, \Psi]\left(\bar{y}_{0}, \bar{y}_{T}\right) .
$$

Let $N_{\pi}(\bar{u}, \bar{y})$ be the set of nonzero $\lambda \in N(\bar{u}, \bar{y})$ having an associated costate. 
We define the set-valued mapping $U:[0, T] \rightrightarrows \mathbb{R}^{m}$ by

$$
U(t):=\operatorname{cl}\left\{u \in \mathbb{R}^{m}: c\left(t, u, \bar{y}_{t}\right)<0\right\} \quad \text { for a.a. } t,
$$

where cl denotes the closure in $\mathbb{R}^{m}$. We can now define two different notions of multipliers.

\section{Definition 2.7.}

(i) We say that $\lambda \in N_{\pi}(\bar{u}, \bar{y})$ is a generalized Lagrange multiplier iff

$$
D_{u} H^{a}\left[p_{t}^{\lambda}, \nu_{t}\right]\left(t, \bar{u}_{t}, \bar{y}_{t}\right)=0 \text { for a.a. } t .
$$

We denote by $\Lambda_{L}(\bar{u}, \bar{y})$ the set of generalized Lagrange multipliers.

(ii) We say that $\lambda \in \Lambda_{L}(\bar{u}, \bar{y})$ is a generalized Pontryagin multiplier iff

$$
H\left[p_{t}^{\lambda}\right]\left(t, \bar{u}_{t}, \bar{y}_{t}\right) \leq H\left[p_{t}^{\lambda}\right]\left(t, u, \bar{y}_{t}\right) \quad \text { for all } u \in U(t), \quad \text { for a.a. } t .
$$

We denote by $\Lambda_{P}(\bar{u}, \bar{y})$ the set of generalized Pontryagin multipliers.

Note that $\Lambda_{L}(\bar{u}, \bar{y})$ and $\Lambda_{P}(\bar{u}, \bar{y})$ are convex cones.

Remark 2.8. The set-valued mapping $U(t)$ describes the feasible controls for the mixed constraints, for the state variable $\bar{y}$. Note that by continuity, for a.a. $t$, the inclusion

$$
U(t) \subset\left\{u \in \mathbb{R}^{m}: c\left(t, u, \bar{y}_{t}\right) \leq 0\right\}
$$

holds but may be strict. In ([2], Appendix), we give an example where this inclusion is strict and where for a.a. $t, \bar{u}_{t}$ minimizes $u \mapsto H\left[p_{t}^{\lambda}\right]\left(t, u, \bar{y}_{t}\right)$ over $U(t)$ but not over the r.h.s. of (2.26) (the Lagrange multiplier $\lambda$ being unique in this example).

Remark 2.9. As a consequence of the inward condition (Assumption 2), we get that

$$
\bar{u}_{t} \in U(t), \quad \text { for a.a. } t .
$$

\subsection{Reducible touch points}

Let us first recall the definition of the order of a state constraint. For $1 \leq i \leq n_{g}$, assuming that $g_{i}$ is sufficiently regular, we define by induction $g_{i}^{(j)}:[0, T] \times \mathbb{R}^{m} \times \mathbb{R}^{n} \rightarrow \mathbb{R}, j \in \mathbb{N}$, by

$$
g_{i}^{(j+1)}(t, u, y):=D_{t} g_{i}^{(j)}(t, u, y)+D_{y} g_{i}^{(j)}(t, u, y) f(t, u, y), \quad g_{i}^{(0)}:=g_{i} .
$$

Definition 2.10. If $g_{i}$ and $f$ are $C^{q_{i}}$, we say that the state constraint $g_{i}$ is of order $q_{i} \in \mathbb{N}$ iff

$$
D_{u} g_{i}^{(j)} \equiv 0 \quad \text { for } 0 \leq j \leq q_{i}-1, \quad D_{u} g_{i}^{\left(q_{i}\right)} \not \equiv 0 .
$$

If $g_{i}$ is of order $q_{i}$, then for all $j<q_{i}, g_{i}^{(j)}$ is independent of $u$ and we do not mention this dependence anymore. Moreover, the mapping $t \mapsto g_{i}\left(t, \bar{y}_{t}\right)$ belongs to $W^{q_{i}, \infty}(0, T)$ and

$$
\begin{gathered}
\frac{\mathrm{d}^{j}}{\mathrm{~d} t^{j}} g_{i}\left(t, \bar{y}_{t}\right)=g_{i}^{(j)}\left(t, \bar{y}_{t}\right) \quad \text { for } 0 \leq j<q_{i}, \\
\frac{\mathrm{d}^{j}}{\mathrm{~d} t^{j}} g_{i}\left(t, \bar{y}_{t}\right)=g_{i}^{(j)}\left(t, \bar{u}_{t}, \bar{y}_{t}\right) \quad \text { for } j=q_{i} .
\end{gathered}
$$


Definition 2.11. We say that $\tau \in[0, T]$ is a touch point for the constraint $g_{i}$ iff it is a contact point for $g_{i}$, i.e. $g_{i}\left(\tau, \bar{y}_{\tau}\right)=0$, and $\tau$ is isolated in $\left\{t: g_{i}\left(t, \bar{y}_{t}\right)=0\right\}$. We say that a touch point $\tau$ for $g_{i}$ is reducible iff $\tau \in(0, T)$, $\frac{\mathrm{d}^{2}}{\mathrm{~d} t^{2}} g_{i}\left(t, \bar{y}_{t}\right)$ is defined for $t$ close to $\tau$, continuous at $\tau$, and

$$
\left.\frac{\mathrm{d}^{2}}{\mathrm{~d} t^{2}} g_{i}\left(t, \bar{y}_{t}\right)\right|_{t=\tau}<0
$$

For $1 \leq i \leq n_{g}$, let us define

$$
\mathcal{T}_{g, i}:= \begin{cases}\emptyset & \text { if } g_{i} \text { is of order } 1 \\ \left\{\text { touch points for } g_{i}\right\} & \text { otherwise }\end{cases}
$$

Note that for the moment, we only need to distinguish the constraints of order 1 from the other constraints, for which the order may be undefined if $g_{i}$ or $f$ is not regular enough.

Assumption 3. For $1 \leq i \leq n_{g}$, the set $\mathcal{T}_{g, i}$ - if nonempty - is finite and only contains reducible touch points.

Note that we do not need supplementary assumptions of the structure of $\left\{t: g_{i}\left(t, \bar{y}_{t}\right)=0\right\}$, in particular, there may be an infinite number of boundary arcs.

\subsection{Tools for the second-order analysis}

We define now the linearizations of the system, the critical cone, and the Hessian of the Lagrangian. Let us set

$$
\mathcal{V}_{2}:=L^{2}\left(0, T ; \mathbb{R}^{m}\right), \quad \mathcal{Z}_{1}:=W^{1,1}\left(0, T ; \mathbb{R}^{n}\right), \quad \text { and } \quad \mathcal{Z}_{2}:=W^{1,2}\left(0, T ; \mathbb{R}^{n}\right) .
$$

Given $v \in \mathcal{V}_{2}$, we consider the linearized state equation in $\mathcal{Z}_{2}$

$$
\dot{z}_{t}=D f\left(t, \bar{u}_{t}, \bar{y}_{t}\right)\left(v_{t}, z_{t}\right) \quad \text { for a.a. } t \in(0, T) .
$$

We call linerarized trajectory any $(v, z) \in \mathcal{V}_{2} \times \mathcal{Z}_{2}$ such that (2.35) holds. For any $\left(v, z^{0}\right) \in \mathcal{V}_{2} \times \mathbb{R}^{n}$, there exists a unique $z \in \mathcal{Z}_{2}$ such that (2.35) holds and $z_{0}=z^{0}$; we denote it by $z=z\left[v, z^{0}\right]$. We also consider the second-order linearized state equation in $\mathcal{Z}_{1}$, defined by

$$
\dot{\zeta}_{t}=D_{y} f\left(t, \bar{u}_{t}, \bar{y}_{t}\right) \zeta_{t}+D^{2} f\left(t, \bar{u}_{t}, \bar{y}_{t}\right)\left(v_{t}, z_{t}\left[v, z^{0}\right]\right)^{2} \quad \text { for a.a. } t \in(0, T) .
$$

We denote by $z^{2}\left[v, z^{0}\right]$ the unique $\zeta \in \mathcal{Z}_{1}$ such that $(2.36)$ holds and such that $\zeta_{0}=0$.

The critical cone in $L^{2}$ is defined by

$$
C_{2}(\bar{u}, \bar{y}):=\left\{\begin{array}{l}
(v, z) \in \mathcal{V}_{2} \times \mathcal{Z}_{2}: z=z\left[v, z_{0}\right] \\
D \phi\left(\bar{y}_{0}, \bar{y}_{T}\right)\left(z_{0}, z_{T}\right) \leq 0 \\
D \Phi\left(\bar{y}_{0}, \bar{y}_{T}\right)\left(z_{0}, z_{T}\right) \in T_{K_{\Phi}}\left(\Phi\left(\bar{y}_{0}, \bar{y}_{T}\right)\right) \\
D c(\cdot, \bar{u}, \bar{y})(v, z) \in T_{K_{c}}(c(\cdot, \bar{u}, \bar{y})) \\
D g(\cdot, \bar{y}) z \in T_{K_{g}}(g(\cdot, \bar{y}))
\end{array}\right\}
$$

Note that by ([6], Examples 2.63 and 2.64), the tangent cones $T_{K_{g}}(g(\cdot, \bar{y}))$ and $T_{K_{c}}(c(\cdot, \bar{u}, \bar{y}))$ are resp. described by

$$
\begin{aligned}
& T_{K_{g}}=\left\{\zeta \in C\left([0, T] ; \mathbb{R}^{n}\right): \forall i, \forall t, g_{i}\left(t, \bar{y}_{t}\right)=0 \Longrightarrow \zeta_{i, t} \leq 0\right\}, \\
& T_{K_{c}}=\left\{w \in L^{2}\left(0, T ; \mathbb{R}^{m}\right): \forall i, \text { for a.a. } t, c_{i}\left(t, \bar{u}_{t}, \bar{y}_{t}\right)=0 \Longrightarrow w_{i, t} \leq 0\right\}
\end{aligned}
$$


Finally, for any $\lambda=(\beta, \Psi, \nu, \mu) \in E$, we define a quadratic form, the Hessian of Lagrangian, $\Omega[\lambda]: \mathcal{V}_{2} \times \mathcal{Z}_{2} \rightarrow$ $\mathbb{R}$ by

$$
\begin{aligned}
\Omega[\lambda](v, z):= & \int_{0}^{T} D^{2} H^{a}\left[p_{t}^{\lambda}, \nu_{t}\right]\left(t, \bar{u}_{t}, \bar{y}_{t}\right)\left(v_{t}, z_{t}\right)^{2} \mathrm{~d} t+D^{2} \Phi[\beta, \Psi]\left(\bar{y}_{0}, \bar{y}_{T}\right)\left(z_{0}, z_{T}\right)^{2} \\
& +\int_{[0, T]}\left(\mathrm{d} \mu_{t} D^{2} g\left(t, \bar{y}_{t}\right)\left(z_{t}\right)^{2}\right)-\sum_{\substack{\tau \in \mathcal{T}_{g, i} \\
1 \leq i \leq n_{g}}} \mu_{i}(\tau) \frac{\left(D g_{i}^{(1)}\left(\tau, \bar{y}_{\tau}\right) z_{\tau}\right)^{2}}{g_{i}^{(2)}\left(\tau, \bar{u}_{\tau}, \bar{y}_{\tau}\right)}
\end{aligned}
$$

where $\mu_{i}(\tau)$ is the measure of the singleton $\{\tau\}$. We justify the terms involving the touch points in $\mathcal{T}_{g, i}$ in the following section.

\section{Reduction of touch points}

We recall in this section the main idea of the reduction technique used for the touch points of state constraints of order greater or equal than 2. Let us mention that this approach was described in ([12], Sect. 3) and used in ([14], Sect. 4), in the case of optimal control problems. As shown in [3], the reduction allows to derive no-gap necessary and sufficient second-order optimality conditions, i.e., the Hessian of the Lagrangian of the reduced problem corresponds to the quadratic form of the necessary conditions. We also prove a strict differentiability property for the mapping associated with the reduction, that will be used in the decomposition principle. Recall that for all $1 \leq i \leq n_{g}$, all touch points of $\mathcal{T}_{g, i}$ are supposed to be reducible (Assumption 3).

Let $\varepsilon>0$ be sufficiently small so that for all $1 \leq i \leq n_{g}$, for all $\tau \in \mathcal{T}_{g, i}$, the time function

$$
t \in[\tau-\varepsilon, \tau+\varepsilon] \mapsto g\left(t, \bar{y}_{t}\right)
$$

is $C^{2}$ and is such that for some $\beta>0, \frac{\mathrm{d}^{2}}{\mathrm{~d} t^{2}} g_{i}\left(t, \bar{y}_{t}\right) \leq-\beta$, for all $t$ in $[\tau-\varepsilon, \tau+\varepsilon]$. From now on, we set for all $i$ and for all $\tau \in \mathcal{T}_{g, i}$

$$
\Delta_{\tau}^{\varepsilon}=[\tau-\varepsilon, \tau+\varepsilon] \quad \text { and } \quad \Delta_{i}^{\varepsilon}=[0, T] \backslash\left\{\cup_{\tau \in \mathcal{T}_{g, i}} \Delta_{\tau}^{\varepsilon}\right\},
$$

and we consider the mapping $\Theta_{\tau}^{\varepsilon}: \mathcal{U} \times \mathbb{R}^{n} \rightarrow \mathbb{R}$ defined by

$$
\Theta_{\tau}^{\varepsilon}\left(u, y^{0}\right):=\max \left\{g_{i}\left(t, y_{t}\right): y=y\left[u, y^{0}\right], t \in \Delta_{\tau}^{\varepsilon}\right\} .
$$

We define the reduced pure constraints as follows:

$$
\text { for all } i \in\left\{1, \ldots, n_{g}\right\},\left\{\begin{array}{c}
g_{i}\left(t, y_{t}\right) \leq 0, \text { for all } t \in \Delta_{i}^{\varepsilon}, \\
\Theta_{\tau}^{\varepsilon}\left(u, y_{0}\right) \leq 0, \text { for all } \tau \in \mathcal{T}_{g, i}
\end{array}\right.
$$

Finally, we consider the following reduced problem, which is an equivalent reformulation of problem $(P)$, in which the pure constraints are replaced by constraint (3.4):

$$
\min _{(u, y) \in \mathcal{U} \times \mathcal{Y}} \phi\left(y_{0}, y_{T}\right) \quad \text { subject to } \quad(2.1), \quad(2.3), \quad(2.5), \text { and }(3.4) .
$$

Now, for all $1 \leq i \leq n_{g}$, consider the mapping $\rho_{i}$ defined by

$$
\rho_{i}: \mu \in \mathcal{M}\left([0, T] ; \mathbb{R}_{+}\right) \mapsto\left(\mu_{\mid \Delta_{i}^{\varepsilon}},(\mu(\tau))_{\tau \in \mathcal{T}_{g, i}}\right) \in \mathcal{M}\left(\Delta_{i}^{\varepsilon} ; \mathbb{R}_{+}\right) \times \mathbb{R}^{\left|\mathcal{T}_{g, i}\right|}
$$

Lemma 3.1. The mapping $\Theta_{\tau}^{\varepsilon}$ is twice Fréchet-differentiable at $\left(\bar{u}, \bar{y}_{0}\right)$ with derivatives

$$
\begin{aligned}
D \Theta_{\tau}^{\varepsilon}\left(\bar{u}, \bar{y}_{0}\right)\left(v, z_{0}\right) & =D g_{i}\left(\tau, \bar{y}_{\tau}\right) z_{\tau}\left[v, z_{0}\right] \\
D^{2} \Theta_{\tau}^{\varepsilon}\left(\bar{u}, \bar{y}_{0}\right)\left(v, z_{0}\right)^{2} & =D^{2} g_{i}\left(\tau, \bar{y}_{\tau}\right)\left(z_{\tau}\left[v, z_{0}\right]\right)^{2}+D g_{i}\left(\tau, \bar{y}_{\tau}\right) z_{\tau}^{2}\left[v, z_{0}\right]-\frac{\left(D g_{i}^{(1)}\left(\tau, \bar{y}_{\tau}\right) z_{\tau}\right)^{2}}{g_{i}^{(2)}\left(\tau, \bar{u}_{\tau}, \bar{y}_{\tau}\right)} .
\end{aligned}
$$


and the following mappings define a bijection between $\Lambda_{L}(\bar{u}, \bar{y})$ and the Lagrange multipliers of problem $\left(P^{\prime}\right)$, resp. between $\Lambda_{P}(\bar{u}, \bar{y})$ and the Pontryagin multipliers of problem $\left(P^{\prime}\right)$ :

$$
\begin{aligned}
& \lambda=(\beta, \Psi, \nu, \mu) \in \Lambda_{L}(\bar{u}, \bar{y}) \mapsto\left(\beta, \Psi, \nu,\left(\rho_{i}\left(\mu^{i}\right)\right)_{1 \leq i \leq n_{g}}\right) \\
& \lambda=(\beta, \Psi, \nu, \mu) \in \Lambda_{P}(\bar{u}, \bar{y}) \mapsto\left(\beta, \Psi, \nu,\left(\rho_{i}\left(\mu^{i}\right)\right)_{1 \leq i \leq n_{g}}\right) .
\end{aligned}
$$

See ([3], Lem. 26) for a proof of this result. Note that the restriction of $\mu_{i}$ to $\Delta_{i}^{\varepsilon}$ is associated with constraint (3.4(i)) and $\left(\mu_{i}(\tau)\right)_{\tau \in \mathcal{T}_{g, i}}$ with constraint (3.4(ii)). The expression of the Hessian of $\Theta_{\tau}^{\varepsilon}$ justifies the quadratic form $\Omega$ defined in (2.40). Note also that in the sequel, we will work with problem $P^{\prime}$ and with the original description of the multipliers, using implicitly the bijections (3.8) and (3.9).

Now, let us fix $i$ and $\tau \in \mathcal{T}_{g, i}$. The following lemma is a differentiability property for the mapping $\Theta_{\tau}^{\varepsilon}$, related to the one of strict differentiability, that will be used to prove the decomposition theorem.

Lemma 3.2. There exists $\varepsilon>0$ such that for all $u_{1}$ and $u_{2}$ in $\mathcal{U}$, for all $y^{0}$ in $\mathbb{R}^{n}$, if

$$
\left\|u^{1}-\bar{u}\right\|_{1} \leq \varepsilon, \quad\left\|u^{2}-\bar{u}\right\|_{1} \leq \varepsilon, \quad \text { and } \quad\left|y^{0}-\bar{y}_{0}\right| \leq \varepsilon,
$$

then

$$
\begin{aligned}
& \Theta_{\tau}^{\varepsilon}\left(u^{2}, y^{0}\right)-\Theta_{\tau}^{\varepsilon}\left(u^{1}, y^{0}\right)=g\left(\tau, y_{\tau}\left[u^{2}, y^{0}\right]\right)-g\left(\tau, y_{\tau}\left[u^{1}, y^{0}\right]\right) \\
& +O\left(\left\|u^{2}-u^{1}\right\|_{1}\left(\left\|u^{1}-\bar{u}\right\|_{1}+\left\|u^{2}-\bar{u}\right\|_{1}+\left|y^{0}-\bar{y}_{0}\right|\right)\right)
\end{aligned}
$$

An intermediate lemma is needed to prove this result. Consider the mapping $\chi$ defined as follows:

$$
\chi: x \in W^{2, \infty}\left(\Delta_{\tau}^{\varepsilon}\right) \mapsto \sup _{t \in[\tau-\varepsilon, \tau+\varepsilon]} x_{t} \in \mathbb{R} .
$$

Let us set $x^{0}=g_{i}(\cdot, \bar{y})_{\mid \Delta_{\tau}^{\varepsilon}}$. Note that $\dot{x}_{\tau}^{0}=0$.

Lemma 3.3. There exists $\alpha^{\prime}>0$ such that for all $x^{1}$ and $x^{2}$ in $W^{2, \infty}\left(\Delta_{\tau}\right)$, if $\left\|\dot{x}^{1}-\dot{x}^{0}\right\|_{\infty} \leq \alpha^{\prime}$ and $\left\|\dot{x}^{2}-\dot{x}^{0}\right\|_{\infty} \leq$ $\alpha^{\prime}$, then

$$
\chi\left(x^{2}\right)-\chi\left(x^{1}\right)=x^{2}(\tau)-x^{1}(\tau)+O\left(\left\|\dot{x}^{2}-\dot{x}^{1}\right\|_{\infty}\left(\left\|\dot{x}^{1}-\dot{x}^{0}\right\|_{\infty}+\left\|\dot{x}^{2}-\dot{x}^{0}\right\|_{\infty}\right)\right) .
$$

Proof. Let $0<\alpha^{\prime}<\beta \varepsilon$ and $x^{1}, x^{2}$ in $W^{2, \infty}\left(\Delta_{\tau}\right)$ satisfy the assumption of the lemma. Denote by $\tau_{1}$ (resp. $\tau_{2}$ ) a (possibly non-unique) maximizer of $\chi\left(x^{1}\right)$ (resp. $\left.\chi\left(x^{2}\right)\right)$. Since

$$
\dot{x}_{\tau-\varepsilon}^{1} \geq \dot{x}_{\tau-\varepsilon}^{0}-\alpha^{\prime} \geq \beta \varepsilon-\alpha^{\prime}>0 \quad \text { and } \quad \dot{x}_{\tau+\varepsilon}^{1} \leq \dot{x}_{\tau+\varepsilon}^{0}+\alpha \leq-\beta \varepsilon+\alpha<0,
$$

we obtain that $\tau_{1} \in(\tau-\varepsilon, \tau+\varepsilon)$ and therefore that $\dot{x}_{\tau_{1}}^{1}=0$. Therefore,

$$
\beta\left|\tau_{1}-\tau\right| \leq\left|\dot{x}_{\tau_{1}}^{0}-\dot{x}_{\tau}^{0}\right|=\left|\dot{x}_{\tau_{1}}^{1}-\dot{x}_{\tau_{1}}^{0}\right| \leq\left\|\dot{x}^{1}-\dot{x}^{0}\right\|_{\infty}
$$

and then, $\left|\tau_{1}-\tau\right| \leq\left\|\dot{x}^{1}-\dot{x}^{0}\right\|_{\infty} / \beta$. Similarly, $\left|\tau_{2}-\tau\right| \leq\left\|\dot{x}^{2}-\dot{x}^{0}\right\|_{\infty} / \beta$. Then, by (3.15),

$$
\begin{aligned}
\chi\left(x^{2}\right) & \geq x^{1}\left(\tau_{1}\right)+\left(x^{2}\left(\tau_{1}\right)-x^{1}\left(\tau_{1}\right)\right) \\
& =\chi\left(x^{1}\right)+\left(x^{2}(\tau)-x^{1}(\tau)\right)+O\left(\left\|\dot{x}^{2}-\dot{x}^{1}\right\|_{\infty}\left|\tau_{1}-\tau\right|\right)
\end{aligned}
$$

and therefore, the l.h.s. of (3.13) is greater than the r.h.s. and by symmetry, the converse inequality holds. The lemma is proved. 
Proof of Lemma 3.2. Consider the mapping

$$
G_{\tau}:\left(u, y^{0}\right) \in\left(\mathcal{U} \times \mathbb{R}^{n}\right) \mapsto\left(t \in \Delta_{\tau} \mapsto g_{i}\left(t, y_{t}\left[u, y^{0}\right]\right)\right) \in W^{2, \infty}\left(\Delta_{\tau}\right) .
$$

Since $g_{i}$ is not of order 1 and by Assumption 1, the mapping $G_{\tau}$ is Lipschitz in the following sense: there exists $K>0$ such that for all $\left(u^{1}, y^{0,1}\right)$ and $\left(u^{2}, y^{0,2}\right)$,

$$
\left\|G_{\tau}\left(u^{1}, y^{0,1}\right)-G_{\tau}\left(u^{2}, y^{0,2}\right)\right\|_{1, \infty} \leq K\left(\left\|u^{2}-u^{1}\right\|_{1}+\left|y^{0,2}-y^{0,1}\right|\right) .
$$

Set $\alpha=\alpha^{\prime} /(2 K)$. Let $u^{1}$ and $u^{2}$ in $\mathcal{U}$, let $y^{0}$ in $\mathbb{R}^{n}$ be such that (3.10) holds. Then by Lemma 3.3 and by (3.18),

$$
\begin{aligned}
\Theta_{\tau}^{\varepsilon}\left(u^{2}, y^{0}\right)-\Theta_{\tau}^{\varepsilon}\left(u^{1}, y^{0}\right)= & \chi\left(G_{\tau}\left(u^{2}, y^{0}\right)\right)-\chi\left(G_{\tau}\left(u^{1}, y^{0}\right)\right) \\
= & g\left(y_{\tau}\left[u^{2}, y^{0}\right]\right)-g\left(y_{\tau}\left[u^{1}, y^{0}\right]\right) \\
& +O\left(\left\|u^{2}-u^{1}\right\|_{1}\left(\left\|u^{2}-\bar{u}\right\|_{1}+\left\|u^{1}-\bar{u}\right\|_{1}+\left|y^{0}-\bar{y}_{0}\right|\right)\right),
\end{aligned}
$$

as was to be proved.

\section{A DECOMPOSITION PRINCIPLE}

We follow a classical approach by contradiction to prove the quadratic growth property for bounded strong solutions. We assume the existence of a sequence of feasible trajectories $\left(u^{k}, y^{k}\right)_{k}$ which is such that $u^{k}$ is bounded and such that $\left\|y^{k}-\bar{y}\right\|_{\infty} \rightarrow 0$ and for which the quadratic growth property does not hold. The Lagrangian function first provides a lower estimate of the cost function $\phi\left(y_{0}^{k}, y_{T}^{k}\right)$. The difficulty here is to linearize the Lagrangian, since we must consider large perturbations of the control in $L^{\infty}$ norm. To that purpose, we extend the decomposition principle of ([5], Sect. 2.4) to our more general framework with pure and mixed constraints. This principle is a partial expansion of the Lagrangian, which is decomposed into two terms: $\Omega[\lambda]\left(v^{A, k}, z\left[v^{A, k}, y_{0}^{k}-\bar{y}_{0}\right]\right)$, where $v^{A, k}$ stands for the small perturbations of the optimal control, and a difference of Hamiltonians where the large perturbations occur.

\subsection{Notations and first estimates}

Let $R>\|\bar{u}\|_{\infty}$, let $\left(u^{k}, y^{k}\right)_{k}$ be a sequence of feasible trajectories such that

$$
\forall k,\left\|u^{k}\right\|_{\infty} \leq R, \quad\left|y_{0}^{k}-\bar{y}_{0}\right| \rightarrow 0, \quad \text { and } \quad\left\|u^{k}-\bar{u}\right\|_{2} \rightarrow 0 .
$$

This sequence will appear in the proof of the quadratic growth property. Note that the convergence of controls and initial values of the state implies that $\left\|y^{k}-\bar{y}\right\|_{\infty} \rightarrow 0$. We need to build two auxiliary controls $\tilde{u}^{k}$ and $u^{A, k}$. The first one, $\tilde{u}^{k}$, is such that

$$
\left\{\begin{array}{l}
c\left(t, \tilde{u}_{t}^{k}, y_{t}^{k}\right) \leq 0, \text { for a.a. } t \in[0, T], \\
\left\|\tilde{u}^{k}-\bar{u}\right\|_{\infty}=O\left(\left\|y^{k}-\bar{y}\right\|_{\infty}\right) .
\end{array}\right.
$$

The following lemma proves the existence of such a control.

Lemma 4.1. There exist $\varepsilon>0$ and $\alpha \geq 0$ such that for all $y \in \mathcal{Y}$ with $\|y-\bar{y}\|_{\infty} \leq \varepsilon$, there exists $u \in \mathcal{U}$ satisfying

$$
\|u-\bar{u}\|_{\infty} \leq \alpha\|y-\bar{y}\|_{\infty} \text { and } c\left(t, u_{t}, y_{t}\right) \leq 0, \text { for a.a. } t .
$$

Proof. For all $y \in \mathcal{Y}$, consider the mapping $C_{y}$ defined by

$$
u \in \mathcal{U} \mapsto C_{y}(u)=\left(t \mapsto c\left(t, u_{t}, y_{t}\right)\right) \in L^{\infty}\left(0, T ; \mathbb{R}^{n_{c}}\right) .
$$

The inward condition (Assumption 2) corresponds to Robinson's constraint qualification for $C_{\bar{y}}$ at $\bar{u}$ with respect to $L^{\infty}\left(0, T ; \mathbb{R}_{-}^{n_{c}}\right)$. Thus, by the Robinson-Ursescu stability Theorem ([6], Thm. 2.87), there exists $\varepsilon>0$ such 
that for all $y \in \mathcal{Y}$ with $\|y-\bar{y}\|_{\infty} \leq \varepsilon, C_{y}$ is metric regular at $\bar{u}$ with respect to $L^{\infty}\left(0, T ; \mathbb{R}_{-}^{n_{c}}\right)$. Therefore, for all $y \in \mathcal{Y}$ with $\|y-\bar{y}\|_{\infty} \leq \varepsilon$, there exists a control $u$ such that, for almost all $t, c\left(t, u_{t}, y_{t}\right) \leq 0$ and

$$
\|u-\bar{u}\|_{\infty}=O\left(\operatorname{dist}\left(C_{y}(\bar{u}), L^{\infty}\left(0, T ; \mathbb{R}_{-}^{n_{c}}\right)\right)\right)=O\left(\|y-\bar{y}\|_{\infty}\right) .
$$

This proves the lemma.

Now, let us introduce the second auxiliary control $u^{A, k}$. We say that a partition $(A, B)$ of the interval $[0, T]$ is measurable iff $A$ and $B$ are measurable subset of $[0, T]$. Let us consider a sequence of measurable partitions $\left(A_{k}, B_{k}\right)_{k}$ of $[0, T]$. We define $u^{A, k}$ as follows:

$$
u_{t}^{A, k}=\bar{u}_{t} \mathbf{1}_{\left\{t \in B^{k}\right\}}+u_{t}^{k} \mathbf{1}_{\left\{t \in A_{k}\right\}} .
$$

The idea is to separate, in the perturbation $u^{k}-\bar{u}$, the small and large perturbations in uniform norm. In the sequel, the letter $A$ will refer to the small perturbations and the letter $B$ to the large ones. The large perturbations will occur on the subset $B_{k}$.

For the sake of clarity, we suppose from now that the following holds:

$$
\left\{\begin{array}{l}
\left(A_{k}, B_{k}\right)_{k} \text { is a sequence of measurable partitions of }[0, T] \\
\left|y_{0}^{k}-\bar{y}_{0}\right|+\left\|u^{A, k}-\bar{u}\right\|_{\infty} \rightarrow 0 \\
\left|B_{k}\right| \rightarrow 0
\end{array}\right.
$$

where $\left|B_{k}\right|$ is the Lebesgue measure of $B_{k}$. We set

$$
v^{A, k}:=u^{A, k}-\bar{u} \quad \text { and } \quad v^{B, k}:=u^{k}-u^{A, k}
$$

and we define

$$
\delta y^{k}:=y^{k}-\bar{y}, \quad y^{A, k}:=y\left[u^{A, k}, y_{0}^{k}\right], \quad \text { and } \quad z^{A, k}:=z\left[v^{A, k}, \delta y_{0}^{k}\right] .
$$

Let us introduce some useful notations for the future estimates:

$$
\begin{array}{ll}
R_{1, k}:=\left\|u^{k}-\bar{u}\right\|_{1}+\left|\delta y_{0}^{k}\right|, & R_{2, k}:=\left\|u^{k}-\bar{u}\right\|_{2}+\left|\delta y_{0}^{k}\right|, \\
R_{1, A, k}:=\left\|v^{A, k}\right\|_{1}+\left|\delta y_{0}^{k}\right|, & R_{2, A, k}:=\left\|v^{A, k}\right\|_{2}+\left|\delta y_{0}^{k}\right|, \\
R_{1, B, k}:=\left\|v^{B, k}\right\|_{1}, & R_{2, B, k}:=\left\|v^{B, k}\right\|_{2} .
\end{array}
$$

Combining the Cauchy-Schwarz inequality and assumption (4.6), we obtain that

$$
R_{1, B, k} \leq R_{2, B, k}\left|B_{k}\right|^{1 / 2}=o\left(R_{2, B, k}\right) .
$$

Note that by Gronwall's lemma,

$$
\left\|\delta y^{k}\right\|_{\infty}=O\left(R_{1, k}\right)=O\left(R_{2, k}\right) \quad \text { and } \quad\left\|z^{A, k}\right\|_{\infty}=O\left(R_{1, A, k}\right)=O\left(R_{2, k}\right) .
$$

Note also that

$$
\left\|\delta y^{k}-\left(y^{A, k}-\bar{y}\right)\right\|_{\infty}=O\left(R_{1, B, k}\right)=o\left(R_{2, k}\right)
$$

and since $\left\|y^{A, k}-\left(\bar{y}+z^{A, k}\right)\right\|_{\infty}=O\left(R_{2, k}^{2}\right)$,

$$
\left\|\delta y^{k}-z^{A, k}\right\|_{\infty}=o\left(R_{2, k}\right) .
$$




\subsection{Result}

We can now state the decomposition principle.

Theorem 4.2. Suppose that Assumptions 1, 2, and 3 hold. Let $R>\|\bar{u}\|_{\infty}$, let $\left(u^{k}, y^{k}\right)_{k}$ be a sequence of feasible controls satisfying (4.1) and $\left(A_{k}, B_{k}\right)_{k}$ satisfy (4.6). Then, for all $\lambda=(\beta, \Psi, \nu, \mu) \in \Lambda_{L}(\bar{u}, \bar{y})$,

$$
\beta\left(\phi\left(y_{0}^{k}, y_{T}^{k}\right)-\phi\left(\bar{y}_{0}, \bar{y}_{T}\right)\right) \geq \frac{1}{2} \Omega[\lambda]\left(v^{A, k}, z^{A, k}\right)+\int_{B_{k}}\left[H\left[p_{t}^{\lambda}\right]\left(t, u_{t}^{k}, \bar{y}_{t}\right)-H\left[p_{t}^{\lambda}\right]\left(t, \tilde{u}_{t}^{k}, \bar{y}_{t}\right)\right] \mathrm{d} t+o\left(R_{2, k}^{2}\right),
$$

where $\Omega$ is defined by $(2.40)$.

The proof is given at the end of the section, Lemma 4.4. The basic idea to obtain a lower estimate of $\beta\left(\phi\left(y_{0}, y_{T}\right)-\phi\left(\bar{y}_{0}, \bar{y}_{T}\right)\right)$ is classical: we dualize the constraints and expand up to the second order the obtained Lagrangian. However, the dualization of the mixed constraint is particular here, in so far as the nonpositive added term is the following:

$$
\int_{A_{k}} \nu_{t}\left(c\left(t, u_{t}^{A, k}, y_{t}^{k}\right)-c\left(t, \bar{u}_{t}, \bar{y}_{t}\right)\right) \mathrm{d} t+\int_{B_{k}} \nu_{t}\left(c\left(t, \tilde{u}_{t}^{k}, y_{t}^{k}\right)-c\left(t, \bar{u}_{t}, \bar{y}_{t}\right)\right) \mathrm{d} t
$$

where $\tilde{u}^{k}$ and $u^{A, k}$ are defined by (4.2) and (4.5). In some sense, we do not dualize the mixed constraint when there are large perturbations of the control. By doing so, we prove that the contribution of the large perturbations is of the same order as the difference of Hamiltonians appearing in (4.14). If we dualized the mixed constraint with the following term:

$$
\int_{0}^{T} \nu_{t}\left(c\left(t, u_{t}^{k}, y_{t}^{k}\right)-c\left(t, \bar{u}_{t}, \bar{y}_{t}\right)\right) \mathrm{d} t
$$

we would obtain for the contribution of large perturbations a difference of augmented Hamiltonians.

Let us fix $\lambda \in \Lambda_{L}(\bar{u}, \bar{y})$ and let us consider the following two terms:

$$
\begin{aligned}
I_{1}^{k}= & \int_{0}^{T}-H_{y}^{a}\left[p_{t}^{\lambda}, \nu_{t}\right]\left(t, \bar{u}_{t}, \bar{y}_{t}\right) \delta y_{t}^{k} \mathrm{~d} t \\
& +\int_{A_{k}}\left(H^{a}\left[p_{t}^{\lambda}, \nu_{t}\right]\left(t, u_{t}^{A, k}, y_{t}^{k}\right)-H^{a}\left[p_{t}^{\lambda}, \nu_{t}\right]\left(t, \bar{u}_{t}, \bar{y}_{t}\right)\right) \mathrm{d} t \\
& +\int_{B_{k}}\left(H^{a}\left[p_{t}^{\lambda}, \nu_{t}\right]\left(t, \tilde{u}_{t}^{k}, y_{t}^{k}\right)-H^{a}\left[p_{t}^{\lambda}, \nu_{t}\right]\left(t, \bar{u}_{t}, \bar{y}_{t}\right)\right) \mathrm{d} t \\
& +\int_{B_{k}}\left(H\left[p_{t}^{\lambda}\right]\left(t, u_{t}^{k}, y_{t}^{k}\right)-H\left[p_{t}^{\lambda}\right]\left(t, \tilde{u}_{t}^{k}, y_{t}^{k}\right)\right) \mathrm{d} t
\end{aligned}
$$

and

$$
\begin{aligned}
I_{2}^{k}= & -\int_{[0, T]}\left(\mathrm{d} \mu_{t} D g\left(t, \bar{y}_{t}\right) \delta y_{t}^{k}\right)+\sum_{i=1}^{n_{g}} \int_{\Delta_{i}^{\varepsilon}}\left(g_{i}\left(t, y_{t}^{k}\right)-g_{i}\left(t, \bar{y}_{t}\right)\right) \mathrm{d} \mu_{i, t} \\
& +\sum_{\substack{\tau \in \mathcal{T}_{g, i} \\
1 \leq i \leq n_{g}}} \mu_{i}(\tau)\left(\Theta_{\tau}^{\varepsilon}\left(u^{k}, y_{0}^{k}\right)-\Theta_{\tau}^{\varepsilon}\left(\bar{u}, \bar{y}_{0}\right)\right) .
\end{aligned}
$$

Lemma 4.3. Let $R>\|\bar{u}\|_{\infty}$, let $\left(u^{k}, y^{k}\right)_{k}$ be a sequence of feasible trajectories satisfying (4.1), and let $\left(A_{k}, B_{k}\right)_{k}$ satisfy (4.6). Then, for all $\lambda \in \Lambda_{L}(\bar{u}, \bar{y})$, the following lower estimate holds:

$$
\beta\left(\phi\left(y_{0}^{k}, y_{T}^{k}\right)-\phi\left(\bar{y}_{0}, \bar{y}_{T}\right)\right) \geq \frac{1}{2} D^{2} \Phi[\beta, \Psi]\left(\bar{y}_{0}, \bar{y}_{T}\right)\left(z_{0}^{A, k}, z_{T}^{A, k}\right)^{2}+I_{1}^{k}+I_{2}^{k}+o\left(R_{2, k}^{2}\right) .
$$


Proof. Let $\lambda \in \Lambda_{L}(\bar{u}, \bar{y})$. In view of sign conditions for constraints and multipliers, we first obtain that

$$
\begin{aligned}
& \beta\left(\phi\left(y_{0}^{k}, y_{T}^{k}\right)-\phi\left(\bar{y}_{0}, \bar{y}_{T}\right)\right) \geq \Phi[\beta, \Psi]\left(y_{0}^{k}, y_{T}^{k}\right)-\Phi[\beta, \Psi]\left(\bar{y}_{0}, \bar{y}_{T}\right) \\
& +\sum_{i=1}^{n_{g}} \int_{\Delta_{i}^{\varepsilon}}\left(g_{i}\left(t, y_{t}^{k}\right)-g_{i}\left(t, \bar{y}_{t}\right)\right) \mathrm{d} \mu_{i, t}+\sum_{\substack{\tau \in \mathcal{T}_{g, i} \\
1 \leq i \leq n_{g}}} \mu_{i}(\tau)\left(\Theta_{\tau}^{\varepsilon}\left(u^{k}, y_{0}^{k}\right)-\Theta_{\tau}^{\varepsilon}\left(\bar{u}, \bar{y}_{0}\right)\right) \\
& +\int_{A_{k}} \nu_{t}\left(c\left(t, u_{t}^{A, k}, y_{t}^{k}\right)-c\left(t, \bar{u}_{t}, \bar{y}_{t}\right)\right) \mathrm{d} t+\int_{B_{k}} \nu_{t}\left(c\left(t, \tilde{u}_{t}^{k}, y_{t}^{k}\right)-c\left(t, \bar{u}_{t}, \bar{y}_{t}\right)\right) \mathrm{d} t .
\end{aligned}
$$

Expanding the end-point Lagrangian up to the second order, and using (4.13), we obtain that

$$
\begin{aligned}
\Phi[\beta, \Psi]\left(y_{0}^{k}, y_{T}^{k}\right)-\Phi[\beta, \Psi]\left(\bar{y}_{0}, \bar{y}_{T}\right) & =D \Phi[\beta, \Psi]\left(\bar{y}_{0}, \bar{y}_{T}\right)\left(\delta y_{0}^{k}, \delta y_{T}^{k}\right)+\frac{1}{2} D^{2} \Phi[\beta, \Psi]\left(\bar{y}_{0}, \bar{y}_{T}\right)\left(\delta y_{0}^{k}, \delta y_{T}^{k}\right)^{2}+o\left(R_{2, k}^{2}\right) \\
& =\left(p_{T}^{\lambda} \delta y_{T}^{k}-p_{0}^{\lambda} \delta y_{0}^{k}\right)+\frac{1}{2} D^{2} \Phi[\lambda]\left(\bar{y}_{0}, \bar{y}_{T}\right)\left(z_{0}^{A, k}, z_{T}^{A, k}\right)^{2}+o\left(R_{2, k}^{2}\right) .
\end{aligned}
$$

Integrating by parts (see [3], Lem. 32), we obtain that

$$
\begin{aligned}
p_{T}^{\lambda} \delta y_{T}^{k}-p_{0}^{\lambda} \delta y_{0}^{k}= & \int_{[0, T]}\left(\mathrm{d} p_{t}^{\lambda} \delta y_{t}^{k}+p_{t}^{\lambda} \dot{\delta} y_{t}^{k} \mathrm{~d} t\right) \\
= & \int_{0}^{T}\left(-D_{y} H^{a}\left[p_{t}^{\lambda}, \nu_{t}\right]\left(t, \bar{u}_{t}, \bar{y}_{t}\right) \delta y_{t}^{k}+H\left[p_{t}^{\lambda}\right]\left(t, u_{t}^{k}, y_{t}^{k}\right)-H\left[p_{t}^{\lambda}\right]\left(t, \bar{u}_{t}, \bar{y}_{t}\right)\right) \mathrm{d} t \\
& -\int_{[0, T]}\left(\mathrm{d} \mu_{t} D g\left(t, \bar{y}_{t}\right) \delta y_{t}^{k}\right) .
\end{aligned}
$$

The lemma follows from (4.20), (4.21), and (4.22).

The following lemma is also a lower estimate of $\beta\left(\phi\left(y_{T}^{k}, y_{0}^{k}\right)-\phi\left(\bar{y}_{T}, \bar{y}_{0}\right)\right)$. It will be used in the proof of the quadratic growth property. Note that the sequence of feasible trajectories which is involved in the lemma does not satisfy (4.1).

Lemma 4.4. Let $\left(u^{k}, y^{k}\right)_{k}$ be a sequence of feasible trajectories. Then, for all $\lambda \in \Lambda_{L}(\bar{u}, \bar{y})$, the following lower estimate holds:

$$
\beta\left(\phi\left(y_{T}^{k}, y_{0}^{k}\right)-\phi\left(\bar{y}_{T}, \bar{y}_{0}\right)\right) \geq \int_{0}^{T}\left(H\left[p_{t}^{\lambda}\right]\left(t, u_{t}^{k}, \bar{y}_{t}\right)-H\left[p_{t}^{\lambda}\right]\left(t, \bar{u}_{t}, \bar{y}_{t}\right)\right) \mathrm{d} t+O\left(\left\|\delta y^{k}\right\|_{\infty}\right) .
$$

Proof. Similarly to the proof of Lemma 4.3, we have that

$$
\begin{aligned}
\beta\left(\phi\left(y_{T}^{k}, y_{0}^{k}\right)-\phi\left(\bar{y}_{T}, \bar{y}_{0}\right)\right) \geq & \Phi[\beta, \Psi]\left(y_{0}^{k}, y_{T}^{k}\right)-\Phi[\beta, \Psi]\left(\bar{y}_{0}, \bar{y}_{T}\right) \\
\geq & \left(p_{T}^{\lambda} \delta y_{T}^{k}-p_{0}^{\lambda} \delta y_{0}^{k}\right)+o\left(\left\|\delta y^{k}\right\|_{\infty}\right) \\
= & \int_{0}^{T}\left(-D_{y} H^{a}\left[p_{t}^{\lambda}, \nu_{t}\right]\left(t, \bar{u}_{t}, \bar{y}_{t}\right) \delta y_{t}^{k}+H\left[p_{t}^{\lambda}\right]\left(t, u_{t}^{k}, y_{t}^{k}\right)-H\left[p_{t}^{\lambda}\right]\left(t, \bar{u}_{t}, \bar{y}_{t}\right)\right) \mathrm{d} t \\
& -\int_{[0, T]}\left(\mathrm{d} \mu_{t} D g\left(t, \bar{y}_{t}\right) \delta y_{t}^{k}\right)+o\left(\left\|\delta y^{k}\right\|_{\infty}\right) .
\end{aligned}
$$

The result follows.

Proof of the decomposition principle. We prove Theorem 4.2 by estimating the two terms $I_{1}^{k}$ and $I_{2}^{k}$ obtained in Lemma 4.3. 
$\triangleright$ Estimation of $I_{1}^{k}$.

Let show that

$$
I_{1}^{k}=\frac{1}{2} \int_{0}^{T} D^{2} H^{a}\left[p_{t}^{\lambda}, \nu_{t}\right]\left(t, \bar{u}_{t}, \bar{y}_{t}\right)\left(v_{t}^{A, k}, z_{t}^{A, k}\right)^{2} \mathrm{~d} t+\int_{B_{k}}\left(H\left[p_{t}^{\lambda}\right]\left(t, u_{t}^{k}, \bar{y}_{t}\right)-H\left[p_{t}^{\lambda}\right]\left(t, \tilde{u}_{t}^{k}, \bar{y}_{t}\right)\right) \mathrm{d} t+o\left(R_{2, k}^{2}\right) .
$$

Using (4.13) and the stationarity of the augmented Hamiltonian, we obtain that term (4.17a) is equal to

$$
\int_{A_{k}} D_{y} H^{a}\left[p_{t}^{\lambda}, \nu_{t}\right]\left(t, \bar{u}_{t}, \bar{y}_{t}\right) \delta y_{t}^{k} \mathrm{~d} t+\frac{1}{2} \int_{A_{k}} D^{2} H^{a}\left[p_{t}^{\lambda}, \nu_{t}\right]\left(t, \bar{u}_{t}, \bar{y}_{t}\right)\left(v_{t}^{A, k}, z_{t}^{A, k}\right)^{2} \mathrm{~d} t+o\left(R_{2, k}^{2}\right) .
$$

Term (4.17b) is negligible compared to $R_{2, k}^{2}$. Since

$\int_{B_{k}}\left(H\left[p_{t}^{\lambda}\right]\left(t, u_{t}^{k}, y_{t}^{k}\right)-H\left[p_{t}^{\lambda}\right]\left(t, \tilde{u}_{t}^{k}, y_{t}^{k}\right)\right) \mathrm{d} t-\int_{B_{k}}\left(H\left[p_{t}^{\lambda}\right]\left(t, u_{t}^{k}, \bar{y}_{t}\right)-H\left[p_{t}^{\lambda}\right]\left(t, \tilde{u}_{t}^{k}, \bar{y}_{t}\right)\right) \mathrm{d} t=O\left(\left|B_{k}\right| R_{1, k}^{2}\right)=o\left(R_{2, k}^{2}\right)$,

term $(4.17 \mathrm{c})$ is equal to

$$
\int_{B_{k}}\left(H\left[p_{t}^{\lambda}\right]\left(t, u_{t}^{k}, \bar{y}_{t}\right)-H\left[p_{t}^{\lambda}\right]\left(t, \tilde{u}_{t}^{k}, \bar{y}_{t}\right)\right) \mathrm{d} t+o\left(R_{2, k}^{2}\right) .
$$

The following term is also negligible:

$$
\int_{B_{k}} D^{2} H^{a}\left[p_{t}^{\lambda}\right]\left(t, \bar{u}_{t}, \bar{y}_{t}\right)\left(v_{t}^{A, k}, z_{t}^{A, k}\right)^{2} \mathrm{~d} t=o\left(R_{2, k}^{2}\right) .
$$

Finally, combining (4.17), (4.27), (4.29), and (4.30), we obtain (4.26).

$\triangleright$ Estimation of $I_{2}^{k}$.

Let us show that

$$
I_{2}^{k}=\frac{1}{2} \int_{[0, T]}\left(\mathrm{d} \mu_{t} D^{2} g\left(t, \bar{y}_{t}\right)\left(z_{t}^{A, k}\right)^{2}\right)-\frac{1}{2} \sum_{\substack{\tau \in \mathcal{T}_{g, i} \\ 1 \leq i \leq n_{g}}} \mu_{i}(\tau) \frac{\left(D g_{i}^{(1)}\left(\tau, \bar{y}_{\tau}\right) z_{\tau}^{A, k}\right)^{2}}{g_{i}^{(2)}\left(\tau, \bar{u}_{\tau}, \bar{y}_{\tau}\right)}
$$

Using (4.13), we obtain the following estimate of term (4.18a):

$$
-\sum_{\substack{\tau \in \mathcal{T}_{g, i} \\ 1 \leq i \leq n_{g}}} \int_{\Delta_{\tau}^{\varepsilon}} D g_{i}\left(t, \bar{y}_{t}\right) \delta y_{t}^{k} \mathrm{~d} \mu_{i, t}+\frac{1}{2} \sum_{i=1}^{n_{g}} \int_{\Delta_{i}^{\varepsilon}} D^{2} g_{i}\left(t, \bar{y}_{t}\right)\left(z_{t}^{A, k}\right)^{2} \mathrm{~d} \mu_{t}+o\left(R_{2, k}^{2}\right) .
$$

Remember that $z^{2}\left[v^{A, k}, \delta y_{0}^{k}\right]$ denotes the second-order linearization (2.36) and that the following holds:

$$
\left\|y^{A, k}-\left(\bar{y}+z\left[v^{A, k}, \delta y_{0}^{k}\right]+z^{2}\left[v^{A, k}, \delta y_{0}^{k}\right]\right)\right\|_{\infty}=o\left(R_{2, k}^{2}\right)
$$

Using Lemma 3.2 and estimate (4.13), we obtain that for all $i$, for all $\tau \in \mathcal{T}_{g, i}$,

$$
\begin{aligned}
\Theta_{\tau}^{\varepsilon}\left(u^{k}, y_{0}^{k}\right)-\Theta_{\tau}^{\varepsilon}\left(u^{A, k}, y_{0}^{k}\right) & =g_{i}\left(\tau, y_{\tau}^{k}\right)-g_{i}\left(\tau, y_{\tau}^{A, k}\right)+O\left(R_{1, B, k}\left(R_{1, B, k}+R_{1, k}\right)\right) \\
& =D g_{i}\left(\tau, \bar{y}_{\tau}\right)\left(y_{\tau}^{k}-y_{\tau}^{A, k}\right)+o\left(R_{2, k}^{2}\right) \\
& =D g_{i}\left(\tau, \bar{y}_{\tau}\right)\left(\delta y_{\tau}^{k}-z_{\tau}^{A, k}-z_{\tau}^{2}\left[v^{A, k}, \delta y_{0}^{k}\right]\right)+o\left(R_{2, k}^{2}\right) .
\end{aligned}
$$

By Lemma 3.1,

$$
\begin{aligned}
\Theta_{\tau}^{\varepsilon}\left(u^{A, k}, y_{0}^{k}\right)-\Theta_{\tau}^{\varepsilon}\left(\bar{u}, \bar{y}_{0}\right)= & D g_{i}\left(\tau, \bar{y}_{\tau}\right)\left(z_{\tau}^{A, k}+z_{\tau}^{2}\left[v^{A, k}, \delta y_{0}^{k}\right]\right) \\
& +\frac{1}{2} D^{2} g_{i}\left(\tau, \bar{y}_{\tau}\right)\left(z_{\tau}^{A, k}\right)^{2}-\frac{1}{2} \frac{\left.\left(D_{y} g_{i}^{(1)}\left(\tau, \bar{y}_{\tau}\right) z_{\tau}^{A, k}\right)^{2}\right)}{g_{i}^{(2)}\left(\tau, \bar{u}_{\tau}, \bar{y}_{\tau}\right)}+o\left(R_{2, k}^{2}\right)
\end{aligned}
$$


Recall that the restriction of $\mu_{i}$ to $\Delta_{\tau}^{\varepsilon}$ is a Dirac measure at $\tau$. Summing (4.34) and (4.35), we obtain the following estimate for $(4.18 \mathrm{~b})$ :

$$
\sum_{\substack{\tau \in \mathcal{T}_{g, i} \\ 1 \leq i \leq n_{g}}}\left[\int_{\Delta_{\tau}^{\varepsilon}}\left(D g_{i}\left(t, \bar{y}_{t}\right) \delta y_{t}^{k}+\frac{1}{2} D^{2} g_{i}\left(t, \bar{y}_{t}\right)\left(z_{t}^{A, k}\right)^{2}\right) \mathrm{d} \mu_{i, t}-\frac{1}{2} \frac{\left.\left(D g_{i}^{(1)}\left(\tau, \bar{y}_{\tau}\right) z_{\tau}^{A, k}\right)^{2}\right)}{g_{i}^{(2)}\left(\tau, \bar{u}_{\tau}, \bar{y}_{\tau}\right)}\right]+o\left(R_{2, k}^{2}\right) .
$$

Combining (4.32) and (4.36), we obtain (4.31). Combining (4.26) and (4.31), we obtain the result.

\section{QUADRATIC GROWTH FOR BOUNDED STRONG SOLUTIONS}

We give in this section sufficient second-order optimality conditions in Pontryagin form ensuring the quadratic growth property for bounded strong solutions. Our main result, Theorem 5.4, is proved with a classical approach by contradiction.

Assumption 4. There exists $\varepsilon>0$ such that for all feasible trajectories $(u, y)$ in $(\mathcal{U} \times \mathcal{Y})$ with $\|y-\bar{y}\| \leq \varepsilon$, if $(u, y)$ satisfies the mixed constraints, then there exists $\hat{u}$ such that

$$
\hat{u}_{t} \in U(t) \text {, for a.a. } t \text { and }\|u-\hat{u}\|_{\infty}=O\left(\|y-\bar{y}\|_{\infty}\right) .
$$

Remark 5.1. This assumption is a metric regularity property, global in $u$ and local in $y$. Note that the required property is different from the property required for $\tilde{u}^{k}(4.2)$.

Definition 5.2. A quadratic form $Q$ on a Hilbert space $X$ is said to be a Legendre form iff it is weakly lower semi-continuous and if it satisfies the following property: if $x^{k} \rightarrow x$ weakly in $X$ and $Q\left(x^{k}\right) \rightarrow Q(x)$, then $x^{k} \rightarrow x$ strongly in $X$.

Assumption 5. For all $\lambda \in \Lambda_{P}(\bar{u}, \bar{y}), \Omega[\lambda]$ is a Legendre form.

Remark 5.3. By ([3], Lem. 21), this assumption is satisfied if for all $\lambda \in \Lambda_{P}(\bar{u}, \bar{y})$, there exists $\gamma>0$ such that for almost all $t$,

$$
\gamma I \leq D_{u u}^{2} H^{a}\left[p_{t}^{\lambda}, \nu_{t}\right]\left(t, \bar{u}_{t}, \bar{y}_{t}\right),
$$

where $I$ is the identity matrix of $\mathbb{R}^{m \times m}$. In particular, in the absence of mixed and control constraints, the quadratic growth of the Hamiltonian (5.4) implies (5.2).

For all $R>\|\bar{u}\|_{\infty}$, we define

$$
\begin{array}{r}
\Lambda_{P}^{R}(\bar{u}, \bar{y})=\left\{\lambda \in \Lambda_{L}(\bar{u}, \bar{y}): \text { for a.a. } t, \text { for all } u \in U(t) \text { with }|u| \leq R,\right. \\
\left.H\left[p_{t}^{\lambda}\right]\left(t, u, \bar{y}_{t}\right)-H\left[p_{t}^{\lambda}\right]\left(t, \bar{u}_{t}, \bar{y}_{t}\right) \geq 0\right\} .
\end{array}
$$

Note that $\Lambda_{P}(\bar{u}, \bar{y})=\cap_{R>\|\bar{u}\|_{\infty}} \Lambda_{P}^{R}(\bar{u}, \bar{y})$. Remember that $C_{2}(\bar{u}, \bar{y})$ is the critical cone in $L^{2}$, defined by $(2.37)$.

Theorem 5.4. Suppose that Assumptions 1-5 hold. If the following second-order sufficient conditions hold: for all $R>\|\bar{u}\|_{\infty}$,

1. there exist $\alpha>0$ and $\lambda^{*} \in \Lambda_{P}^{R}(\bar{u}, \bar{y})$ such that

$$
\left\{\begin{array}{l}
\text { for a.a.t for all } u \in U(t) \text { with }|u| \leq R \\
H\left[p_{t}^{\lambda^{*}}\right]\left(t, u, \bar{y}_{t}\right)-H\left[p_{t}^{\lambda^{*}}\right]\left(t, \bar{u}_{t}, \bar{y}_{t}\right) \geq \alpha\left|u-\bar{u}_{t}\right|^{2}
\end{array}\right.
$$

2. for all $(v, z) \in C_{2} \backslash\{0\}$, there exists $\lambda \in \Lambda_{P}^{R}(\bar{u}, \bar{y})$ such that $\Omega[\lambda](v, z)>0$, then the quadratic growth property for bounded strong solutions holds at $(\bar{u}, \bar{y})$. 
Proof. We prove this theorem by contradiction. Let $R>\|\bar{u}\|_{\infty}$, let $R^{\prime}>R$, let us suppose that there exists a sequence $\left(u^{k}, y^{k}\right)_{k}$ of feasible trajectories such that $\left\|u^{k}\right\|_{\infty} \leq R,\left\|y^{k}-\bar{y}\right\|_{\infty} \rightarrow 0$ and

$$
\phi\left(y_{0}^{k}, y_{T}^{k}\right)-\phi\left(\bar{y}_{0}, \bar{y}_{T}\right) \leq o\left(\left\|u^{k}-\bar{u}\right\|_{2}^{2}+\left|y_{0}^{k}-\bar{y}_{0}\right|^{2}\right) .
$$

We use the notations introduced in (4.9). Let $\lambda^{*}=\left(\beta^{*}, \Psi^{*}, \nu^{*}, \mu^{*}\right) \in \Lambda_{P}^{R^{\prime}}(\bar{u}, \bar{y})$ be such that (5.4) holds.

$\triangleright$ First step: $\left\|u^{k}-\bar{u}\right\|_{2}=R_{2, k} \rightarrow 0$.

By Assumption 4, there exists a sequence of controls $\left(\hat{u}^{k}\right)_{k}$ such that

$$
\hat{u}_{t}^{k} \in U(t) \text {, for a.a. } t \text { and }\left\|u^{k}-\hat{u}^{k}\right\|_{\infty}=O\left(\left\|\delta y^{k}\right\|_{\infty}\right)=O\left(R_{1, k}\right) .
$$

As a consequence of (4.23), we obtain that

$$
\begin{aligned}
\beta^{*}\left(\phi\left(y_{0}^{k}, y_{T}^{k}\right)-\phi\left(\bar{y}_{0}, \bar{y}_{T}\right)\right) \geq & \int_{0}^{T}\left(H\left[p_{t}^{\lambda^{*}}\right]\left(t, u_{t}^{k}, \bar{y}_{t}\right)-H\left[p_{t}^{\lambda^{*}}\right]\left(t, \hat{u}_{t}^{k}, \bar{y}_{t}\right)\right) \mathrm{d} t \\
& +\int_{0}^{T}\left(H\left[p_{t}^{\lambda^{*}}\right]\left(t, \hat{u}_{t}^{k}, \bar{y}_{t}\right)-H\left[p_{t}^{\lambda^{*}}\right]\left(t, \bar{u}_{t}, \bar{y}_{t}\right)\right) \mathrm{d} t+o(1) \\
\geq & \alpha\left\|\hat{u}^{k}-\bar{u}\right\|_{2}^{2}+o(1) \\
= & \alpha\left\|u^{k}-\bar{u}\right\|_{2}^{2}+o(1) .
\end{aligned}
$$

Since $\beta^{*}\left(\phi\left(y_{0}^{k}, y_{T}^{k}\right)-\phi\left(\bar{y}_{0}, \bar{y}_{T}\right)\right) \rightarrow 0$, we obtain that $\left\|u^{k}-\bar{u}\right\|_{2} \rightarrow 0$. Therefore, the sequence of trajectories satisfy (4.1) and by the Cauchy-Schwarz inequality, $R_{1, k} \rightarrow 0$.

Now, we can build a sequence of partitions $\left(A_{k}, B_{k}\right)_{k}$ which satisfies (4.6). Let us define

$$
A_{k}:=\left\{t \in[0, T],\left|u_{t}^{k}-\bar{u}_{t}\right| \leq R_{1, k}^{1 / 4}\right\}
$$

and $B_{k}:=[0, T] \backslash A_{k}$. Then,

$$
\left\|u^{k}-\bar{u}\right\|_{1} \geq \int_{B_{k}}\left(\left\|u^{k}-\bar{u}\right\|_{1}+\left|\delta y_{0}^{k}\right|\right)^{1 / 4} \mathrm{~d} t \geq\left|B_{k}\right|\left(\left\|u^{k}-\bar{u}\right\|_{1}\right)^{1 / 4}
$$

Thus, $\left|B_{k}\right| \leq\left(\left\|u^{k}-\bar{u}\right\|_{1}\right)^{3 / 4} \rightarrow 0$ and we can construct all the elements useful for the decomposition principle: $\tilde{u}^{k}, u^{A, k}, v^{A, k}, \delta y^{k}, y^{A, k}$, and $z^{A, k}$.

Let $\bar{\lambda} \in \Lambda_{P}^{R^{\prime}}(\bar{u}, \bar{y}), \sigma \in[0,1)$ and $\lambda:=\sigma \bar{\lambda}+(1-\sigma) \lambda^{*}$. The Hamiltonian depending linearly on the dual variable, the quadratic growth property (5.4) holds for $\lambda$ (instead of $\lambda^{*}$ ) with the coefficient $(1-\sigma) \alpha>0$ (instead of $\alpha$ ).

$\triangleright$ Second step: we show that $R_{2, B, k}=O\left(R_{2, A, k}\right)$ and $\Omega[\lambda]\left(v^{A, k}, z^{A, k}\right) \leq o\left(R_{2, A, k}^{2}\right)$.

By the decomposition principle (Thm. 4.2), we obtain that

$$
\Omega[\lambda]\left(v^{A, k}, z^{A, k}\right)+\int_{B_{k}}\left[H\left[p_{t}^{\lambda}\right]\left(t, u_{t}^{k}, \bar{y}_{t}\right)-H\left[p_{t}^{\lambda}\right]\left(t, \tilde{u}_{t}^{k}, \bar{y}_{t}\right)\right] \mathrm{d} t \leq \beta\left(\phi\left(y_{0}^{k}, y_{T}^{k}\right)-\phi\left(\bar{y}_{0}, \bar{y}_{T}\right)\right)+o\left(R_{2, k}^{2}\right) \leq o\left(R_{2, k}^{2}\right) .
$$

We cannot use directly the quadratic growth of the Hamiltonian, since the control $u^{k}$ does not satisfy necessarily the mixed constraint $c\left(t, u_{t}^{k}, \bar{y}_{t}\right) \leq 0$. Therefore, we decompose the difference of Hamiltonians as follows:

$$
\Delta_{k}=\int_{B_{k}}\left(H\left[p_{t}^{\lambda}\right]\left(t, u_{t}^{k}, \bar{y}_{t}\right)-H\left[p_{t}^{\lambda}\right]\left(t, \tilde{u}_{t}^{k}, \bar{y}_{t}\right)\right) \mathrm{d} t=\Delta_{k}^{a}+\Delta_{k}^{b}+\Delta_{k}^{c}
$$


with

$$
\begin{aligned}
\Delta_{k}^{a} & :=\int_{B_{k}}\left(H\left[p_{t}^{\lambda}\right]\left(t, u_{t}^{k}, \bar{y}_{t}\right)-H\left[p_{t}^{\lambda}\right]\left(t, \hat{u}^{k}, \bar{y}_{t}\right)\right) \mathrm{d} t, \\
\Delta_{k}^{b} & :=\int_{B_{k}}\left(H\left[p_{t}^{\lambda}\right]\left(t, \hat{u}^{k}, \bar{y}_{t}\right)-H\left[p_{t}^{\lambda}\right]\left(t, \bar{u}_{t}, \bar{y}_{t}\right)\right) \mathrm{d} t, \\
\Delta_{k}^{c} & :=\int_{B_{k}}\left(H\left[p_{t}^{\lambda}\right]\left(t, \bar{u}_{t}, \bar{y}_{t}\right)-H\left[p_{t}^{\lambda}\right]\left(t, \tilde{u}_{t}, \bar{y}_{t}\right)\right) \mathrm{d} t .
\end{aligned}
$$

Note that by $(5.9), \Delta_{k} \leq O\left(R_{2, A, k}^{2}\right)+o\left(R_{2, B, k}^{2}\right)$. We set

$$
\hat{R}_{2, B, k}=\left[\int_{B_{k}}\left|\hat{u}_{t}^{k}-\bar{u}_{t}\right|^{2} \mathrm{~d} t\right]^{1 / 2} .
$$

By (5.6), for large values of $k,\left\|\hat{u}^{k}\right\|_{\infty} \leq R^{\prime}$ and therefore, $\Delta_{k}^{b} \geq 0$. In order to prove that $R_{2, B, k}=O\left(R_{2, A, k}\right)$, we need the following two estimates:

$$
\begin{aligned}
& \left|\Delta_{k}^{a}\right|+\left|\Delta_{k}^{c}\right|=o\left(\Delta_{k}^{b}\right), \\
& \left|R_{2, B, k}^{2}-\hat{R}_{2, B, k}^{2}\right|=o\left(R_{2, B, k}^{2}\right) .
\end{aligned}
$$

Since the control is uniformly bounded, the Hamiltonian is Lipschitz with respect to $u$ and we obtain that

$$
\left|\Delta_{k}^{a}\right|+\left|\Delta_{k}^{c}\right|=O\left(\left|B_{k}\right| R_{1, k}\right),
$$

while, as a consequence of the quadratic growth of the Hamiltonian,

$$
\begin{aligned}
\Delta_{k}^{b} & \geq \alpha(1-\sigma) \hat{R}_{2, B, k}^{2} \\
& \geq \alpha(1-\sigma)\left|B_{k}\right|\left(R_{1, k}^{1 / 4}+O\left(R_{1, k}\right)\right)^{2} \\
& \geq \alpha(1-\sigma)\left|B_{k}\right| R_{1, k}^{1 / 2}\left(1+O\left(R_{1, k}^{3 / 4}\right)\right)^{2}
\end{aligned}
$$

which proves (5.12). Combined with (5.9) and $\Omega[\lambda]\left(v^{A, k}, z^{A, k}\right)=O\left(R_{2, A, k}^{2}\right)$, we obtain that

$$
\Delta_{k}^{b}=O\left(\Delta_{k}^{a}+\Delta_{k}^{b}+\Delta_{k}^{c}\right)=O\left(\Delta_{k}\right)=O\left(R_{2, A, k}^{2}\right)+o\left(R_{2, B, k}^{2}\right)
$$

and

$$
\hat{R}_{2, B, k}^{2} \leq \frac{1}{\alpha(1-\sigma)} \Delta_{k}^{b}=O\left(\Delta_{k}\right) \leq O\left(R_{2, A, k}^{2}\right)+o\left(R_{2, B, k}^{2}\right) .
$$

Let us prove (5.13). For all $k$, we have

$$
\begin{aligned}
\left|R_{2, B, k}^{2}-\hat{R}_{2, B, k}^{2}\right| & =\left|\int_{B_{k}}\left(\left|u_{t}^{k}-\bar{u}_{t}\right|^{2}-\left|\hat{u}_{t}^{k}-\bar{u}_{t}^{2}\right|\right) \mathrm{d} t\right| \\
& \leq \int_{B_{k}}\left|u_{t}^{k}-\hat{u}_{t}^{k}\right|\left(\left|u_{t}^{k}-\hat{u}^{k}\right|+2\left|u_{t}^{k}-\bar{u}_{t}\right|\right) \mathrm{d} t \\
& \leq\left\|u^{k}-\hat{u}^{k}\right\|_{\infty}\left(\int_{B_{k}}\left|u_{t}^{k}-\hat{u}_{t}^{k}\right| \mathrm{d} t+2 \int_{B_{k}}\left|u_{t}^{k}-\bar{u}_{t}\right| \mathrm{d} t\right) \\
& =O\left(R_{1, k}\right)\left(O\left(\left|B_{k}\right| R_{1, k}\right)+O\left(R_{1, B, k}\right)\right) \\
& =o\left(R_{2, k}^{2}\right)
\end{aligned}
$$


which proves (5.13), by using (5.15). Combined with (5.17), it follows that

$$
R_{2, B, k}^{2}=\hat{R}_{2, B, k}^{2}+o\left(R_{2, k}^{2}\right)=O\left(R_{2, A, k}^{2}\right)+o\left(R_{2, B, k}^{2}\right)
$$

and finally that

$$
R_{2, B, k}^{2}=O\left(R_{2, A, k}^{2}\right) \quad \text { and } \quad R_{2, k}=O\left(R_{2, A, k}\right) .
$$

Moreover, since $\Delta_{k}^{b} \geq 0$ and by (5.12), (5.16), and (5.19),

$$
\Omega[\lambda]\left(v^{A, k}, z^{A, k}\right) \leq o\left(R_{2, k}^{2}\right)-\Delta_{k}^{a}-\Delta_{k}^{c} \leq o\left(R_{2, k}^{2}\right)+o\left(\Delta^{k}\right) \leq o\left(R_{2, A, k}^{2}\right) .
$$

$\triangleright$ Third step: contradiction.

Let us set

$$
w^{k}=\frac{v^{A, k}}{R_{2, A, k}} \quad \text { and } \quad x^{k}=\frac{z^{A, k}}{R_{2, A, k}}=z\left[w^{k}, \delta y_{0}^{k} / R_{2, A, k}\right] .
$$

The sequence $\left(w^{k}, x_{0}^{k}\right)_{k}$ being bounded in $L^{2}\left(0, T ; \mathbb{R}^{m}\right) \times \mathbb{R}^{n}$, it converges (up to a subsequence) for the weak topology to a limit point, say $\left(w, x_{0}\right)$. Let us set $x=z\left[w, x_{0}\right]$. Let us prove that $(w, x) \in C_{2}(\bar{u}, \bar{y})$. It follows from the continuity of the linear mapping

$$
z:\left(v, z_{0}\right) \in L^{2}\left(0, T ; \mathbb{R}^{m}\right) \times \mathbb{R}^{n} \rightarrow z\left[v, z_{0}\right] \in W^{1,2}\left(0, T ; \mathbb{R}^{n}\right)
$$

and the compact imbedding of $W^{1,2}\left(0, T ; \mathbb{R}^{n}\right)$ into $C\left([0, T] ; \mathbb{R}^{n}\right)$ that extracting if necessary, $\left(x^{k}\right)_{k}$ converges uniformly to $x$. Using (4.13), we obtain that

$$
\begin{aligned}
\left\|\delta y^{k}-R_{2, A, k} x\right\|_{\infty} & =\left\|z^{A, k}-R_{2, A, k} x\right\|_{\infty}+o\left(R_{2, A, k}\right) \\
& =R_{2, A, k}\left(\left\|x^{k}-x\right\|_{\infty}+o(1)\right) \\
& =o\left(R_{2, A, k}\right)
\end{aligned}
$$

It follows that

$$
\begin{aligned}
& \phi\left(y_{0}^{k}, y_{T}^{k}\right)-\phi\left(\bar{y}_{0}, \bar{y}_{T}\right)=R_{2, A, k} D \phi\left(\bar{y}_{0}, \bar{y}_{T}\right)\left(x_{0}, x_{T}\right)+o\left(R_{2, A, k}\right), \\
& \Phi\left(y_{0}^{k}, y_{T}^{k}\right)-\Phi\left(\bar{y}_{0}, \bar{y}_{T}\right)=R_{2, A, k} D \phi\left(\bar{y}_{0}, \bar{y}_{T}\right)\left(x_{0}, x_{T}\right)+o\left(R_{2, A, k}\right), \\
& \left\|g\left(t, y_{t}^{k}\right)-g\left(t, \bar{y}_{t}\right)-R_{2, A, k} D g\left(t, \bar{y}_{t}\right) x_{t}\right\|_{\infty}=o\left(R_{2, A, k}\right) .
\end{aligned}
$$

This proves that

$$
\begin{aligned}
& D \phi\left(\bar{y}_{0}, \bar{y}_{T}\right)\left(x_{0}, x_{T}\right)=0, \\
& D \Phi\left(\bar{y}_{0}, \bar{y}_{T}\right)\left(x_{0}, x_{T}\right) \in T_{K_{\Phi}}\left(\phi\left(\bar{y}_{0}, \bar{y}_{T}\right)\right), \\
& D g(\cdot, \bar{y}) x \in T_{K_{g}}(g(\cdot, \bar{y})) .
\end{aligned}
$$

Let us set, for a.a. $t$,

$$
\bar{c}_{t}=c\left(t, \bar{u}_{t}, \bar{y}_{t}\right) \quad \text { and } \quad c_{t}^{k}=\bar{c}_{t} \mathbf{1}_{\left\{t \in B_{k}\right\}}+c\left(t, u^{A, k}, y_{t}^{k}\right) \mathbf{1}_{\left\{t \in A_{k}\right\}} \text {. }
$$

We easily check that

$$
\left\|c_{t}^{k}-\left(\bar{c}_{t}+R_{2, A, k} D c\left(t, \bar{u}_{t}, \bar{y}_{t}\right)\left(w_{t}^{k}, x_{t}^{k}\right)\right)\right\|_{\infty}=o\left(R_{2, A, k}\right)
$$

Therefore,

$$
\frac{c^{k}-\bar{c}}{R_{2, A, k}} \rightarrow D c\left(t, \bar{u}_{t}, \bar{y}_{t}\right)\left(w_{t}, x_{t}\right)
$$


in $L^{2}\left(0, T ; \mathbb{R}^{n_{c}}\right)$. Moreover, $c_{t}^{k} \leq 0$, for almost all $t$, therefore the ratio in $(5.32)$ belongs to $T_{K_{c}}(c(\cdot, \bar{u}, \bar{y}))$. This cone being closed and convex, it is weakly closed and we obtain finally that

$$
D c\left(t, \bar{u}_{t}, \bar{y}_{t}\right)\left(w_{t}, x_{t}\right) \in T_{K_{c}}(c(\cdot, \bar{u}, \bar{y})) .
$$

We have proved that $(w, x) \in C_{2}(\bar{u}, \bar{y})$. By Assumption $5, \Omega[\lambda]$ is weakly* lower semi-continuous, thus from (5.20), we get that

$$
\Omega[\lambda](w, x) \leq \liminf _{k} \Omega[\lambda]\left(w^{k}, x^{k}\right) \leq 0 .
$$

Passing to the limit when $\sigma \rightarrow 1$, we find that $\Omega[\bar{\lambda}](w, x) \leq 0$. Since $\bar{\lambda}$ was arbitrary in $\Lambda_{P}^{R}(\bar{u}, \bar{y})$, it follows by the sufficient conditions that $\left(w, x_{0}\right)=0$ and that for any $\lambda$ for which the quadratic growth of the Hamiltonian holds,

$$
\Omega[\lambda](w, x)=\lim _{k} \Omega[\lambda]\left(w^{k}, x^{k}\right) .
$$

Since $\Omega[\lambda]$ is a Legendre form, we obtain that $\left(w^{k}, x^{k}\right)_{k}$ converges strongly to 0 , in contradiction with the fact that $\left\|w^{k}\right\|_{2}+\left|x_{0}^{k}\right|=1$. This concludes the proof of the theorem.

\section{Characterization of quadratic Growth}

In this section, we prove that the second-order sufficient conditions are also necessary to ensure the quadratic growth property. The proof relies on the necessary second-order optimality conditions in Pontryagin form that we established in [2]. Let us first remember the assumptions required to use these necessary conditions.

Assumption 6. The mappings $f$ and $g$ are $C^{\infty}, c$ is uniformly quasi- $C^{2}, \Phi$ and $\phi$ are $C^{2}$.

For $\delta^{\prime}>0$ and $\varepsilon^{\prime}>0$, let us define

$$
\begin{aligned}
& \Delta_{c, i}^{\delta^{\prime}}:=\left\{t \in[0, T]: c_{i}\left(t, \bar{u}_{t}, \bar{y}_{t}\right) \geq-\delta^{\prime}\right\}, \\
& \Delta_{g, i}^{0}:=\left\{t \in[0, T]: g_{i}\left(t, \bar{y}_{t}\right)=0\right\} \backslash \mathcal{T}_{g, i}, \\
& \Delta_{g, i}^{\varepsilon^{\prime}}:=\left\{t \in[0, T]: \operatorname{dist}\left(t, \Delta_{g, i}^{0}\right) \leq \varepsilon^{\prime}\right\} .
\end{aligned}
$$

Assumption 7 is a geometrical assumption on the structure of the control. Assumption 8 is related to the controllability of the system, see ([4], Lem. 2.3) for conditions ensuring this property.

Assumption 7. For $1 \leq i \leq n_{g}, \Delta_{g, i}^{0}$ has finitely many connected components and $g_{i}$ is of finite order $q_{i}$.

Assumption 8. There exist $\delta^{\prime}, \varepsilon^{\prime}>0$ such that the linear mapping from $\mathcal{V}_{2} \times \mathbb{R}^{n}$ to $\prod_{i=1}^{n_{c}} L^{2}\left(\Delta_{c, i}^{\delta^{\prime}}\right) \times$ $\prod_{i=1}^{n_{g}} W^{q_{i}, 2}\left(\Delta_{g, i}^{\varepsilon^{\prime}}\right)$ defined by

$$
\left(v, z^{0}\right) \mapsto\left(\begin{array}{c}
\left(\left.D c_{i}(\cdot, \bar{u}, \bar{y})\left(v, z\left[v, z^{0}\right]\right)\right|_{\Delta_{c, i}^{\delta^{\prime}}}\right)_{1 \leq i \leq n_{c}} \\
\left(\left.D g_{i}(\cdot, \bar{y}) z\left[v, z^{0}\right]\right|_{\Delta_{g, i}^{\varepsilon^{\prime}}}\right)_{1 \leq i \leq n_{g}}
\end{array}\right) \quad \text { is onto. }
$$

The second-order necessary conditions are satisfied on a subset of the critical cone called strict critical cone. The following assumption ensures that the two cones are equal ([6], Prop. 3.10).

Assumption 9. There exists $\lambda=(\bar{\beta}, \bar{\Psi}, \bar{\nu}, \bar{\mu}) \in \Lambda_{L}(\bar{u}, \bar{y})$ such that

$$
\begin{array}{ll}
\bar{\nu}_{i}(t)>0 \quad \text { for a.a. } t \in \Delta_{c, i}^{0} & 1 \leq i \leq n_{c}, \\
\operatorname{supp}\left(\bar{\mu}_{i}\right) \cap \Delta_{g, i}^{0}=\Delta_{g, i}^{0} & 1 \leq i \leq n_{g} .
\end{array}
$$


The main result of [2] was the following necessary conditions in Pontryagin form:

Theorem 6.1. Let Assumptions 2, 3, and 6-9 hold. If $(\bar{u}, \bar{y})$ is a Pontryagin minimum of problem $(P)$, then for any $(v, z) \in C_{2}(\bar{u}, \bar{y})$, there exists $\lambda \in \Lambda_{P}(\bar{u}, \bar{y})$ such that

$$
\Omega[\lambda](v, z) \geq 0 .
$$

Assumption 10. All Pontryagin multipliers $\lambda=(\beta, \Psi, \nu, \mu)$ are non singular, that is to say, are such that $\beta>0$.

This assumption is satisfied if one of the usual qualification conditions holds since then, all Lagrange multipliers are non singular. In ([2], Prop. A.13), we gave a weaker condition ensuring the non singularity of Pontryagin multipliers.

Lemma 6.2. Let Assumptions 2, 3, and 6-10 hold. If the quadratic growth property for bounded strong solutions holds at $(\bar{u}, \bar{y})$, then the sufficient second-order conditions are satisfied.

Proof. Let $R>\|\bar{u}\|_{\infty}$, let $\alpha>0$ and $\varepsilon>0$ be such that for all $(u, y) \in F(P)$ with $\|u\|_{\infty} \leq R$ and $\|y-\bar{y}\|_{\infty} \leq \varepsilon$,

$$
\phi\left(y_{0}, y_{T}\right)-\phi\left(\bar{y}_{0}, \bar{y}_{T}\right) \geq \alpha\left(\|u-\bar{u}\|_{2}^{2}+\left|y_{0}-\bar{y}_{0}\right|^{2}\right) .
$$

Then, $(\bar{u}, \bar{y})$ is a Pontryagin minimum to a new optimal control problem with cost

$$
\phi\left(y_{0}, y_{T}\right)-\alpha\left(\left|y_{0}-\bar{y}_{0}\right|^{2}+\|u-\bar{u}\|_{2}^{2}\right)
$$

and with the additional constraint $\|u\|_{\infty} \leq R$. The new Hamiltonian and the new Hessian of the Lagrangian are now given by resp.

$$
H[p](t, u, y)-\alpha \beta|u-\bar{u}|^{2} \quad \text { and } \quad \Omega[\lambda](v, z)-\alpha \beta\left(\|v\|_{2}^{2}+\left|z_{0}\right|^{2}\right) .
$$

It is easy to check that the costate equation is unchanged and that the set of Lagrange multipliers of both problems are the same. The set of Pontryagin multipliers to the new problem is the set of Lagrange multipliers $\lambda$ for which for a.a. $t$, for all $u \in U(t)$ with $|u| \leq R$,

$$
H\left[p_{t}^{\lambda}\right]\left(t, u, \bar{y}_{t}\right)-H\left[p_{t}^{\lambda}\right]\left(t, \bar{u}_{t}, \bar{y}_{t}\right) \geq \alpha \beta|u-\bar{u}|^{2},
$$

it is thus included into $\Lambda_{P}^{R}(\bar{u}, \bar{y})$ (which was defined by $(5.3)$ ). Let $(v, z)$ in $C_{2}(\bar{u}, \bar{y}) \backslash\{0\}$, then by Theorem 6.1 , there exists a Pontryagin multiplier (to the new problem), belonging to $\Lambda_{P}^{R}(\bar{u}, \bar{y})$, which is such that

$$
\Omega[\lambda](v, z) \geq \alpha \beta\left(\left|z_{0}\right|^{2}+\|v\|_{2}^{2}\right)>0 .
$$

The sufficient second-order optimality conditions are satisfied.

Finally, combining Theorem 5.4 and Lemma 6.2 we obtain a characterization of the quadratic growth property for bounded strong solutions (under the Legendre-Clebsch assumption).

Theorem 6.3. Let Assumptions 2-10 hold. Then, the quadratic growth property for bounded strong solutions holds at $(\bar{u}, \bar{y})$ if and only if the sufficient second-order conditions are satisfied.

Acknowledgements. We would like to thank the anonymous referee for his careful reading and valuable comments on our article. 


\section{REFERENCES}

[1] L. Ambrosio, N. Fusco and D. Pallara, Functions of bounded variation and free discontinuity problems. Oxford Mathematical Monographs. The Clarendon Press Oxford University Press, New York (2000).

[2] J.F. Bonnans, X. Dupuis and L. Pfeiffer, Second-order necessary conditions in Pontryagin form for optimal control problems. Inria Research Report RR-8306. INRIA (2013).

[3] J.F. Bonnans and A. Hermant, No-gap second-order optimality conditions for optimal control problems with a single state constraint and control. Math. Program. B 117 (2009) 21-50.

[4] J.F. Bonnans and A. Hermant, Second-order analysis for optimal control problems with pure state constraints and mixed control-state constraints. Ann. Inst. Henri Poincaré Anal. Non Linéaire 26 (2009) 561-598.

[5] J.F. Bonnans and N.P. Osmolovskiǔ, Second-order analysis of optimal control problems with control and initial-final state constraints. J. Convex Anal. 17 (2010) 885-913.

[6] J.F. Bonnans and A. Shapiro, Perturbation analysis of optimization problems. Springer Series in Operations Research. Springer-Verlag, New York (2000).

[7] R. Cominetti, Metric regularity, tangent sets, and second-order optimality conditions. Appl. Math. Opt. 21 (1990) $265-287$.

[8] A.V. Dmitruk, Maximum principle for the general optimal control problem with phase and regular mixed constraints. Software and models of systems analysis. Optimal control of dynamical systems. Comput. Math. Model. 4 (1993) 364-377.

[9] A.Ja. Dubovickiǔ and A.A. Miljutin, Extremal problems with constraints. Ž. Vyčisl. Mat. i Mat. Fiz. 5 (1965) $395-453$.

[10] A.Ja. Dubovickiı̌ and A.A. Miljutin, Necessary conditions for a weak extremum in optimal control problems with mixed constraints of inequality type. Z̆. Vyčisl. Mat. i Mat. Fiz. 8 (1968) 725-779.

[11] M.R. Hestenes, Calculus of variations and optimal control theory. John Wiley \& Sons Inc., New York (1966).

[12] R.P. Hettich and H.Th. Jongen, Semi-infinite programming: conditions of optimality and applications, in Optimization techniques, Proc. 8th IFIP Conf., Würzburg 1977, Part 2. Vol. 7 of Lecture Notes in Control and Information Sci. Springer, Berlin (1978) 1-11.

[13] H. Kawasaki, An envelope-like effect of infinitely many inequality constraints on second-order necessary conditions for minimization problems. Math. Program. 41 (Ser. A) (1988) 73-96.

[14] K. Malanowski and H. Maurer, Sensitivity analysis for optimal control problems subject to higher order state constraints. Optimization with data perturbations II. Ann. Oper. Res. 101 (2001) 43-73.

[15] H. Mäurer, First and second order sufficient optimality conditions in mathematical programming and optimal control. Math. Program. Oberwolfach Proc. Conf. Math. Forschungsinstitut, Oberwolfach (1979). Math. Programming Stud. 14 (1981) 163177.

[16] A.A. Milyutin and N.P. Osmolovskii, Calculus of variations and optimal control, vol. 180 of Translations of Mathematical Monographs. American Mathematical Society, Providence, RI (1998).

[17] N.P. Osmolovskii, Quadratic extremality conditions for broken extremals in the general problem of the calculus of variations. Optimal control and dynamical systems. J. Math. Sci. 123 (2004) 3987-4122.

[18] N.P. Osmolovskii, Sufficient quadratic conditions of extremum for discontinuous controls in optimal control problems with mixed constraints. J. Math. Sci., 173 (2011) 1-106.

[19] N.P. Osmolovskii, Second-order sufficient optimality conditions for control problems with linearly independent gradients of control constraints. ESAIM: COCV 18 (2012) 452-482.

[20] N.P. Osmolovskii and H. Maurer, Applications to regular and bang-bang control, vol. 24 of Advances in Design and Control. Society for Industrial and Applied Mathematics (SIAM), Philadelphia, PA (2012).

[21] L.S. Pontryagin, V.G. Boltyanskii, R.V. Gamkrelidze and E.F. Mishchenko, The Mathematical Theory of Optimal Processes. Translated from the Russian by K.N. Trirogoff, edited by L.W. Neustadt. Interscience Publishers John Wiley \& Sons, Inc. New York-London (1962).

[22] G. Stefani and P. Zezza, Optimality conditions for a constrained control problem. SIAM J. Control Optim 34 (1996) 635-659. 